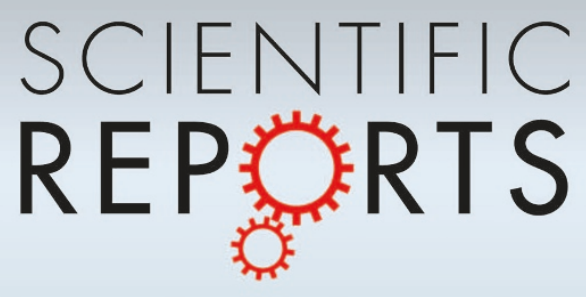

\title{
OPEN A systematic review and meta-analysis of Baihui (GV20)-based scalp acupuncture in experimental ischemic stroke
}

SUBJECT AREAS:

PRE-CLINICAL STUDIES

TRANSLATIONAL RESEARCH

Received

27 November 2013

Accepted

15 January 2014

Published

5 February 2014

Correspondence and requests for materials should be addressed to

G.-Q.Z. Igq_zheng@ sohu.com)

\author{
Wen-wen Wang*, Cheng-long Xie*, Lin Lu \& Guo-qing Zheng
}

Department of Neurology, the Second Affiliated Hospital of Wenzhou Medical University, Wenzhou 325027, China.

Acupuncture for stroke has been used in China for over 2,000 years and nowadays is increasingly practiced elsewhere in the world. However, previous studies had conflicting findings on the results of acupuncture. Here, we conducted a systematic review and meta-analysis to assess the current evidence for the effect of Baihui (GV20)-based scalp acupuncture in animal models of focal cerebral ischemia. Six databases from the inception of each database up to June 2013 were electronically searched. Primary outcomes were infarct size and neurobehavioral outcome. Ultimately, 54 studies involving 1816 animals were identified describing procedures. Meta-analysis results showed that twelve studies reported significant effects of Baihui (GV20)-based scalp acupuncture for improving infarct volume compared with middle cerebral artery occlusion group $(\mathrm{P}<0.01)$, and thirty-two studies reported significant effects of Baihui (GV20)-based scalp acupuncture for improving the neurological function score when compared with the control group (P $<$ 0.01). In conclusion, Baihui (GV20)-based scalp acupuncture could improve infarct volume and neurological function score and exert potential neuroprotective role in experimental ischemic stroke.

* These authors contributed equally to this work.

cupuncture is a therapeutic form of traditional Chinese medicine (TCM) that involves the insertion of fine needles or sometimes laser on the defined points, and usually follows by stimulation using related manual or electrical techniques ${ }^{1}$. Acupuncture has been used for healthcare in China and elsewhere for over 2000 years and now is still a useful medical modality for the treatment of various health problems such as stroke rehabilitation, as recommended by National Institutes of Health consensus panel ${ }^{2}$. Since ancient times, stroke has been very common and is a serious neurological disorder in China. Huangdineijing (Huangdi's Internal Classic), the oldest and greatest extant classic TCM literature written by various unknown authors from the Warring States Period to the Han Dynasty (475 BC-220 AD), first recorded different stroke-related symptoms ${ }^{3}$ and established the theoretical basis for TCM acupuncture therapy. In modern time, acupuncture continued to be widely used for stroke $^{4}$ because stroke remains one of the leading causes of mortality and disability worldwide ${ }^{5}$ and the relative poverty of effective conventional treatments, except intravenous recombinant-tissue plasminogen activator (rtPA) within 4.5 hours after stroke onset ${ }^{6}$.

Baihui (GV20) is an acupoint of the Du meridian (the government vessel), which locates at the intersection of the line connecting the apexes of the two auricles and the median line of the head, 7 cun directly above the posterior hairline and 5 cun behind the anterior hairline according to the TCM theory of acupuncture and the WHO definition ${ }^{7}$. Based on the TCM theory, because Baihui is located on the highest place of the head where all the yang meridians meet ${ }^{8}$, acupuncture on Baihui (GV 20) could clear the mind, lift the spirits, tonify yang, strengthen the ascending function of the spleen, eliminate interior wind, and promote resuscitation ${ }^{9}$. Thus, the acupoint Baihui (GV 20) is specifically used in neurological and psychiatric diseases such as stroke, headache, dizziness, and anxiety. In fact, Baihui (GV 20) is a principle acupoint which is often selected for stroke patients. From a historical perspective, the long history of acupuncture on Baihui (GV 20) for stroke treatment can be traced back to ancient China. During Tang Dynasty, Beiji Qianjin Yaofang (Essential Prescriptions Worth a Thousand Gold for Emergencies), written by Sun Simiao in 652, recorded moxibustion on Baihui (GV 20) for the stroke patients who presented with paralysis and aphasia. During Ming Dynasty, Puji Fang (Prescriptions for Universal Relief), compiled by Zhu Su in 1406, used Baihui (GV 20) acupoint for various symptoms, signs and on different stages of stroke. Especially, scalp acupuncture was set up and separated from traditional acupuncture system under the influence of neuroanatomy, neurophysiology, and bioholographic principle of modern medicine in the early $1970{ }^{\prime} \mathrm{s}^{10}$. Scalp acupuncture is one of the several specialized acupuncture techniques in which a filiform needle is used to penetrate specific stimulation areas on the scalp mainly for the treatment of brain 
diseases ${ }^{10}$. There are various nomenclature systems for scalp acupoint, for example, some divided the scalp into zones or regions, while others focused on points or lines. Baihui (GV20)-based scalp penetration needling, such as needling through Baihui (GV 20) to Taiyang (EX-HN 5) ${ }^{11}$ and needling through Baihui (GV 20) to Qubin $(\mathrm{GB} 7)^{12}$, is one of most important school of scalp acupuncture for stroke. In modern time, various studies have revealed that Baihui (GV20)-based scalp acupuncture has neuroprotective effects on multi-aspects of the pathophysiology in animal models of ischemic stroke. The effects are listed as follows: (1) Scalp acupuncture at Baihui (GV20) and Qubin (GB7) has rapid and powerful effects on removing limb paralyses caused either by cerebral infarct or by cerebral haemorrhage in stroke-prone spontaneously hypertensive rat $^{13}$. (2) Electroacupuncture (EA) preconditioning at Baihui (GV20) could attenuate brain edema and blood brain barrier (BBB) disruption caused by cerebral ischemia and was mainly through decreasing matrix metalloproteinase (MMP)- 9 expression and activity ${ }^{14}$. (3) EA at Baihui (GV20) and Dazhui (GV14) could increase cerebral blood flow and improve tissue and function recovery through acetylcholine/endothelial nitric oxide synthase (eNOS)-mediated perfusion augmentation in acute moderate focal cerebral ischemia ${ }^{15}$. (4) EA pretreatment at Baihui (GV 20) strongly protects the brain against transient cerebral ischemic injury through the anti-inflammatory effects of $\alpha 7$ nicotinic acetylcholine receptors $(\alpha 7 \mathrm{nAChR})$ activation $^{16}$. (5) EA at Baihui(GV20) and Qubin(GB7) could activate the cerebral structures related to motor function on the bilateral hemispheres by assessment of Positron emission tomography (PET) in stroke patients, suggesting that EA was very helpful for the cerebral motor plasticity after the ischemic stroke ${ }^{17}$. (6) EA at Zusanli(ST36) and Baihui(GV20) enhanced cell proliferation and neuroblast differentiation in the rat dentate gyrus via phosphorylated cyclic AMP response element-binding protein ( $\mathrm{PCREB}$ ) and brain-derived neurotrophic factor (BDNF) activation ${ }^{18}$.

In the past decade, several systematic reviews ${ }^{19-25}$ assessing the effects of both acupuncture and scalp acupuncture on patients suffering from stroke have been published; however, the results are inconclusive. Park J and Sze FK et $\mathrm{al}^{19,20}$ reported that acupuncture had no additional effect on motor recovery but had a small positive effect on disability; Kong et $\mathrm{al}^{22}$ pointed out that acupuncture did not show a positive effect as a treatment for functional recovery after stroke from rigorous randomized sham-controlled trials (RCT). On the contrary, Wu et $\mathrm{al}^{23}$ reported that acupuncture was effective in the treatment of post-stroke rehabilitation based on fifty-six RCTs. By comparison, systematic reviews of pre-clinical animal data could inform the planning and improve the likelihood of success of future clinical trials, identify where there is a need for further 'basic' research, preclude unnecessary study replication, and contribute to both 'reduction' and 'refinement' in animal experimentation ${ }^{26}$. Therefore, we conducted a systematic review and meta-analysis of Baihui(GV20)-based Scalp acupuncture (BBA) in animal models of acute focal ischemic stroke.

\section{Results}

Study inclusion. We identified 5383 potentially relevant articles from six databases. After removal of duplicates, 2527 records remained. After going through the titles and abstracts, we excluded 2319 papers with at least one of following reasons: (1) case report or review; (2) not an animal research; and (3) not the researches about stroke or ischemia. By reading the full text of the remaining 208 articles which reported the efficacy of BBA in animal models of focal cerebral ischemia, 54 studies were excluded because the outcome measure was neither neurological function score (NFS) nor infarct volume (IV); 48 studies were excluded because other forms of acupuncture or TCM were used in control group; 34 were excluded because of combination with body acupunctures; 18 studies were removed due to the deficiency of useful data and the problem of duplicate publication. Ultimately, 54 eligible studies were identified

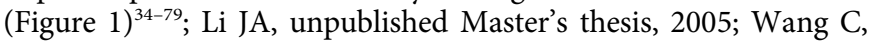
unpublished Master's thesis, 2005; Jin JF, unpublished $\mathrm{PhD}$ thesis, 2005; Luo T, unpublished Master's thesis, 2006; Chen X, unpublished Master's thesis, 2007; Ding J, unpublished Master's thesis, 2007; Wang EL, unpublished PhD thesis, 2008; Wu HY, unpublished $\mathrm{PhD}$ thesis, 2010.

Study characteristics. The 54 included studies with 1816 rats described 73 groups of comparisons based on two different outcome measures: 24 groups of comparisons including 478 rats reported data as IV, and 49 groups of comparisons using 1736 animals reported data as NFS. The rats species included Sprague-Dawley (SD) rats, Wistar rats and stroke-prone spontaneously hypertensive (SHR-SP) rats. The weight of rats varied between 180-340 g (median $240 \mathrm{~g})$. Sixteen out of the 54 studies $(29.6 \%, \mathrm{n}=681)$ were permanent middle cerebral artery occlusion (MCAO) models, while the remaining studies $(n=1135)$ utilized temporary MCAO models. The time of ischemia varied from 30 minutes to 3 hours. Chloral hydrate were used in 32 studies $(n=59.2 \%)$ to induce anesthesia, isoflurane in 7 studies $^{34-36,42,45,57,71}$, Pentobarbital in 9 studies $^{38-41,43,44,46,47,60}$, urethane u $^{59}$ and thiopental ${ }^{69}$ in one study respectively, while no report of anesthetics in the remaining 4 studies $^{66,67,78,79}$.

Eight studies performed manual acupuncture $(\mathrm{MA})^{61,64-67,70,77}$; Wang EL, unpublished $\mathrm{PhD}$ thesis, 2008 and the rest of studies utilized EA. The selection of acupoints were as follows: 12 studies used Baihui mono-therapy ${ }^{34-45} ; 12$ studies selected Baihui plus Shuigou ${ }^{58-63}$; Li JA, unpublished Master's thesis, 2005; Wang C, unpublished Master's thesis, 2005; Jin JF, unpublished $\mathrm{PhD}$ thesis, 2005; Luo T, unpublished Master's thesis, 2006; Chen X, unpublished Master's thesis, 2007; Ding J, unpublished Master's thesis, 2007; 12 studies selected Baihui plus Dazhui ${ }^{46-57} ; 7$ studies used scalp penetration needling through Baihui to other acupoints ${ }^{64-70}$; the rest of the 11 studies selected Baihui plus other acupoints. Meanwhile, IV was used as the outcome measure in 24 groups (44.4\%), and NFS was used in forty-nine groups $(90.7 \%)$. But the standards of NFS were different, as 21 studies adopted Zea long criterion ${ }^{30} ; 13$ studies used Bederson criterion $^{29} ; 10$ studies adopted Garcia criterion ${ }^{31} ; \mathrm{Kuluz}^{80}$, Ludmia $^{81}$, $\mathrm{Cai}^{82}$ and Sun criteria ${ }^{83}$ were cited in 1 study respectively. Sixteen studies adopted both two outcome measures. The basic characteristics of the 54 studies are shown in Table 1.

Study quality and publication bias. The median number of study quality checklist items scored was ranged from 3 to 7 out of a total 10 points. Of whom, seventeen studies got 3 points; sixteen studies got 4 ; ten studies got 5; six studies got 6; and five studies got 7 points (Table 2). Eight studies were online Master's thesis or $\mathrm{PhD}$ thesis and not formally published; Li JA, unpublished Master's thesis, 2005; Wang C, unpublished Master's thesis, 2005; Jin JF, unpublished PhD thesis, 2005; Luo T, unpublished Master's thesis, 2006; Chen X, unpublished Master's thesis, 2007; Ding J, unpublished Master's thesis, 2007; Wang EL, unpublished PhD thesis, 2008; Wu HY, unpublished $\mathrm{PhD}$ thesis, 2010. Twenty-eight studies described control of temperature, including control of the room and rats anal temperature. No study described the sample size calculation. SHR-SP rats were used in one study. Random allocation to treatment group and blinded assessment of outcome were described in 49 and 15 studies respectively. No study reported cerebral ischemia was induced by an investigator who was blinded to treatment allocation. Twenty studies mentioned statement of potential conflict of interests. Twenty-four studies reported compliance with animal welfare regulations. No study used anesthetic with significant intrinsic neuroprotective activity.

Effectiveness. Twelve studies reported significant effects of BBA for improving IV compared with MCAO group $(\mathrm{n}=172$, SMD -1.95 , 95\% CI: $-2.80 \sim-1.10, \mathrm{P}<0.00001$; heterogeneity $\chi^{2}=43.79, \mathrm{P}<$ 


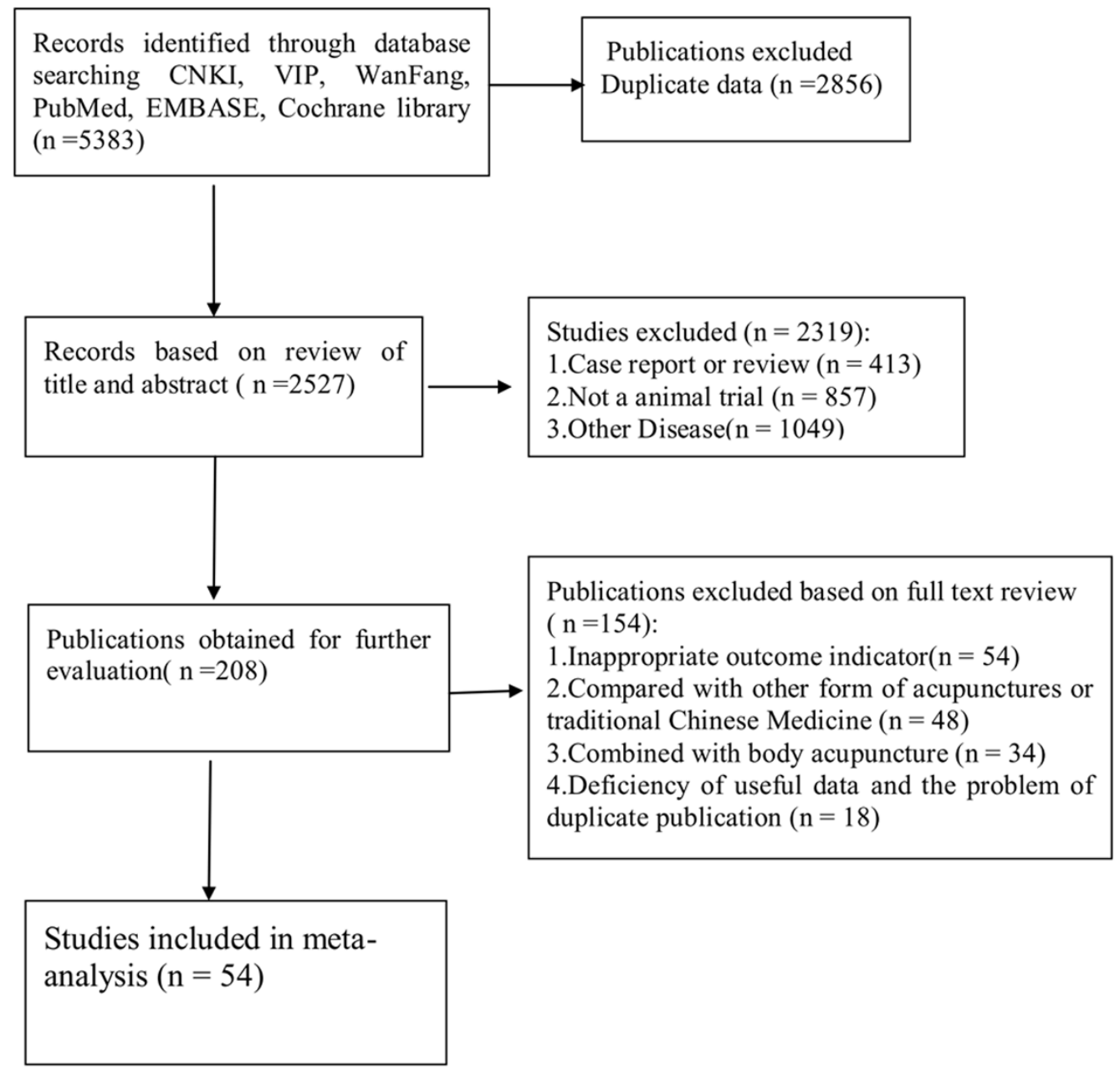

Figure $1 \mid$ The Preferred Reporting Items for Systematic Reviews and Meta-Analyses (PRISMA) Flow Diagram.

$0.00001, \mathrm{I}^{2}=75 \%$, Figure 2); the remaining twelve studies failed to pool analysis due to the data demonstrated in the form of the TTC staining or IV percentage (\%), but all of them reported the significant effects of BBA for ameliorating the IV compared with the control group $(\mathrm{p}<0.05$ or $\mathrm{p}<0.01)$.

Fourteen studies showed significant effects of BBA for improving the NFS according to Zea longa criterion $(\mathrm{n}=425$, SMD $-3.93,95 \%$ CI: $-5.11 \sim-2.75, \mathrm{P}<0.00001$; heterogeneity $\chi 2=179.39, \mathrm{p}<$ $0.00001, I^{2}=93 \%$, Figure 3 ), but the remaining seven studies did not provide raw data and thus failed for meta-analysis. NFS was significantly improved in fourteen studies in BBA group compared with control group according to Bederson criterion $(\mathrm{n}=384, \mathrm{SMD}-1.51$, 95\% CI: $-1.97 \sim-1.05, \mathrm{P}<0.00001$; heterogeneity $\chi 2=44.90, \mathrm{p}<$ $0.00001, I^{2}=71 \%$, Figure 4 ). Four studies reported significant effects of BBA for improving the NFS according to Garcia criterion compared with control group ( $\mathrm{n}=58$, SMD 3.98, 95\% CI: $2.98 \sim 4.98, \mathrm{P}$ $<0.00001$; heterogeneity $\chi 2=0.51, \mathrm{p}=0.92, \mathrm{I}^{2}=0 \%$, Figure 5). Six studies reported significant effects of BBA for improving NFS based on the Garcia criterion $(\mathrm{p}<0.05$ or $\mathrm{p}<0.01$ ), but the data were represented in the graphical form and meta-analysis was unable to be done because we failed to contact the authors. Four studies also reported the significant effects of BBA for ameliorating the NFS according to the Kuluz criteria, Ludmia criteria, Cai criteria, and Sun criteria, respectively ( $\mathrm{p}<0.05$ or $\mathrm{p}<0.01$ ).

Assessment of bias. The funnel plot was roughly symmetric for the effect of BBA on IV and NFS. Thus, funnel plots did not suggest an obvious publication bias (Figure 6).
Factors affecting the outcome measures. In the subgroup analysis for the outcome measure according to NFS, the efficacy of acupuncture at MCAO $30 \mathrm{~min} /$ perfusion was better than other longer perfusion time $(\mathrm{n}=111, \mathrm{SMD}-3.08,95 \% \mathrm{CI}:-3.65 \sim-2.51, \mathrm{P}$ $<0.00001$; heterogeneity $\chi^{2}=2.10, \mathrm{p}=0.35, \mathrm{I}^{2}=5 \%$, Figure 7A). On the other hand, BBA on MCAO 3 hours and permanent model were less effective than on MCAO 1 and 2 hours $(p<0.01)$. Experiments using chloral hydrate as anesthetics showed better improvement of NFS when compared with other anesthetics such as isoflurane and unreported $(\mathrm{n}=291, \mathrm{SMD}-5.02,95 \% \mathrm{CI}:-6.77$ $\sim-3.28, \mathrm{P}<0.00001$; heterogeneity $\chi^{2}=142.99, \mathrm{p}<0.00001, \mathrm{I}^{2}=$ $94 \%$, Figure 7B). Compared with Wistar rats, SD rats were more sensitive to BBA treatment for improving NFS $(\mathrm{n}=214$, SMD $-1.90,95 \% \mathrm{CI}:-2.24 \sim-1.57, \mathrm{P}<0.00001$; heterogeneity $\chi^{2}=$ $9.02, \mathrm{p}=0.25, \mathrm{I}^{2}=22 \%$, Figure 7C). BBA treatment in the published studies was more effective than that in un-published studies $(\mathrm{p}<$ 0.00001, SMD $-3.51,95 \% \mathrm{CI}:-4.07 \sim-2.94 ; \mathrm{p}<0.00001, \mathrm{SMD}$ $-2.40,95 \%$ CI: $-3.02 \sim-1.78$; respectively, Figure 7D). In the subgroup analysis for the outcome measure according to IV, efficacy of BBA at permanent model was better than at other MCAO models (SMD $-2.86,95 \% \mathrm{CI}:-4.12 \sim-1.61, \mathrm{P}<0.00001$; heterogeneity $\chi^{2}=0.95, \mathrm{p}=0.62, \mathrm{I}^{2}=0 \%$, Figure 8A). Experiments using chloral hydrate as anesthetics showed more reduction in IV when compared with those using isoflurane and pentobarbital anesthesia (SMD $-2.59,95 \% \mathrm{CI}:-3.95 \sim-1.21, \mathrm{P}=0.0002$; heterogeneity $\chi 2=$ $21.95, \mathrm{p}=0.0005, \mathrm{I}^{2}=77 \%$, Figure $\left.8 \mathrm{~B}\right)$. Meanwhile, experiments on $\mathrm{SD}$ rats gave a higher estimate of effect size in IV than that using Wistar rats $(\mathrm{SMD}-2.14,95 \% \mathrm{CI}:-3.36 \sim-0.93, \mathrm{P}=0.0005$; 


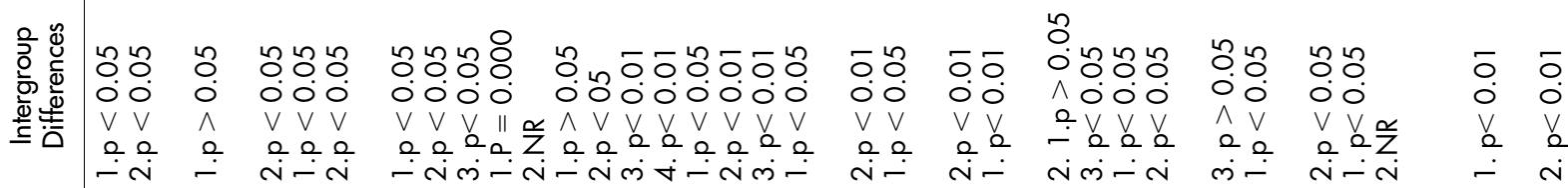

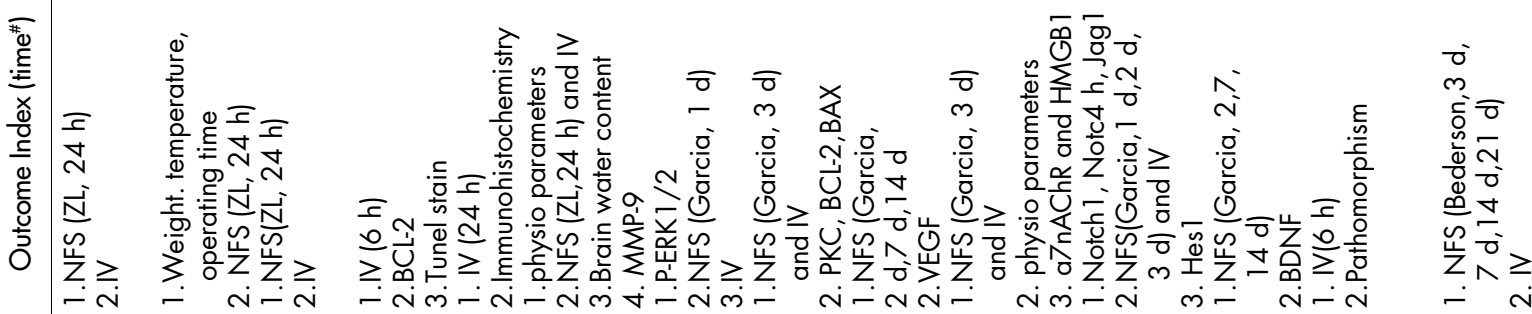

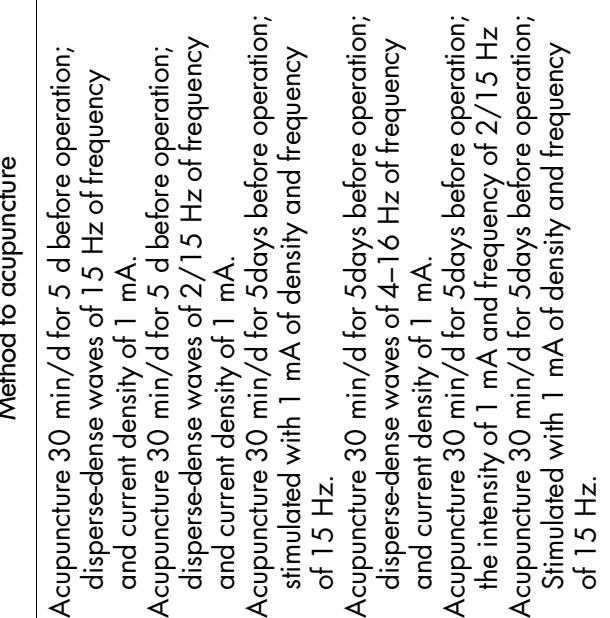

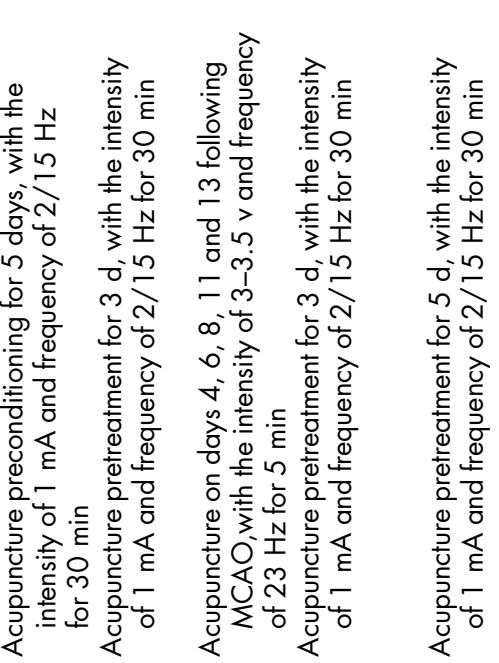

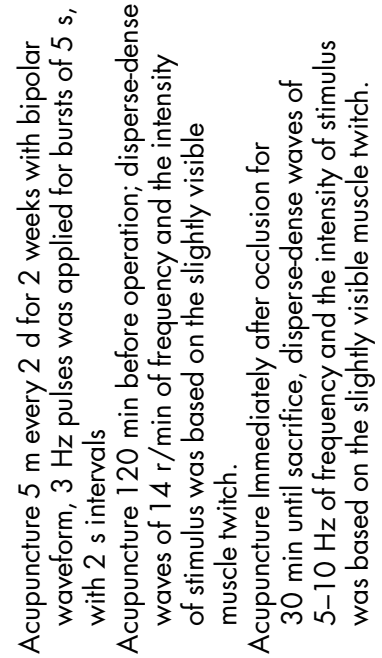
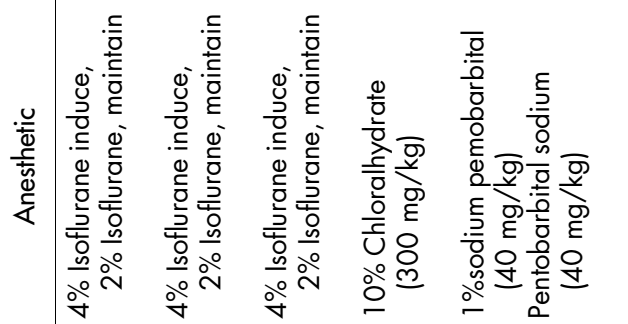

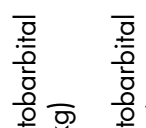
要蕰 豪昌

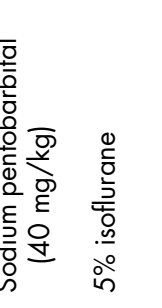

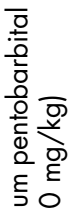
章守

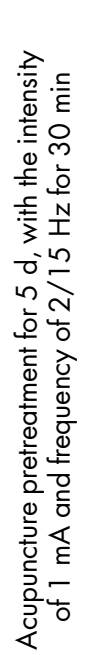

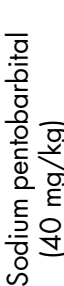

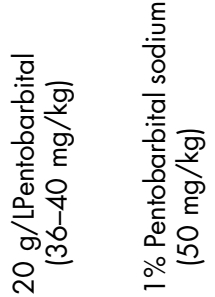

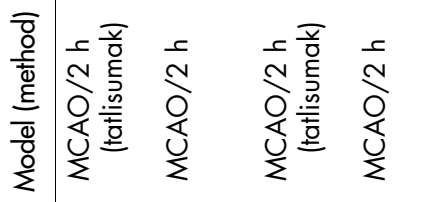

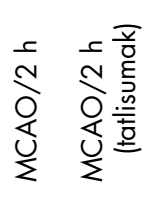

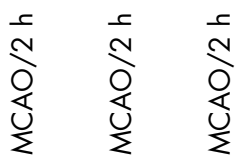

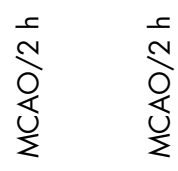

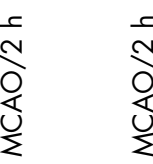

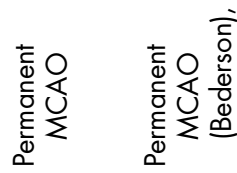

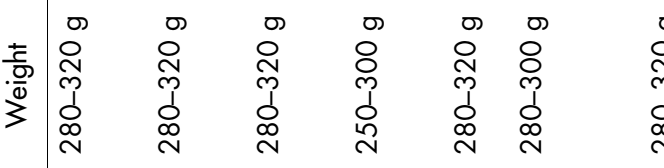

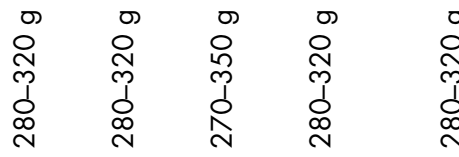

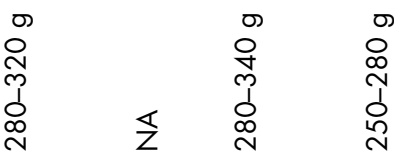

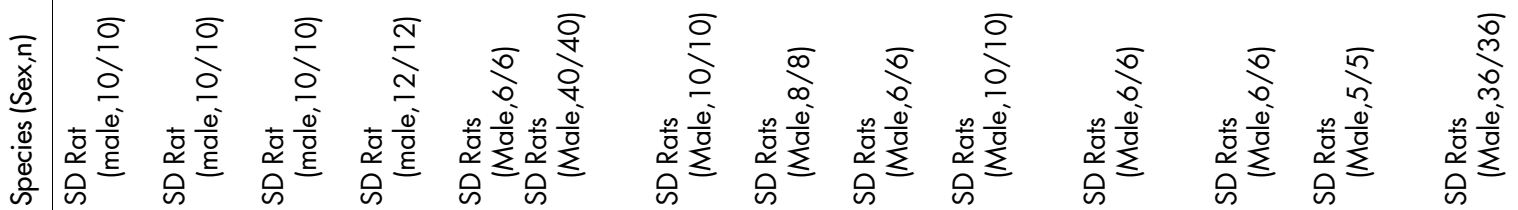

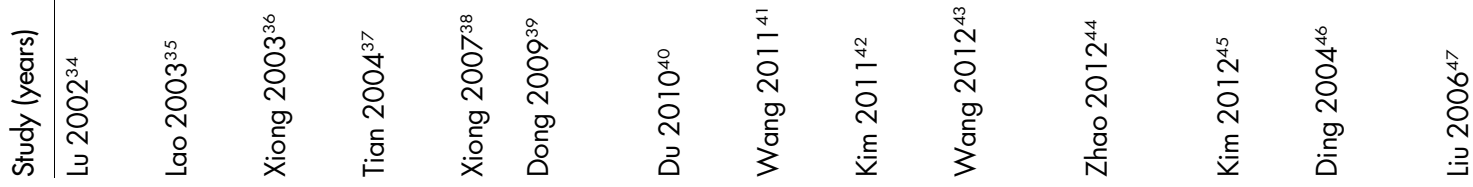




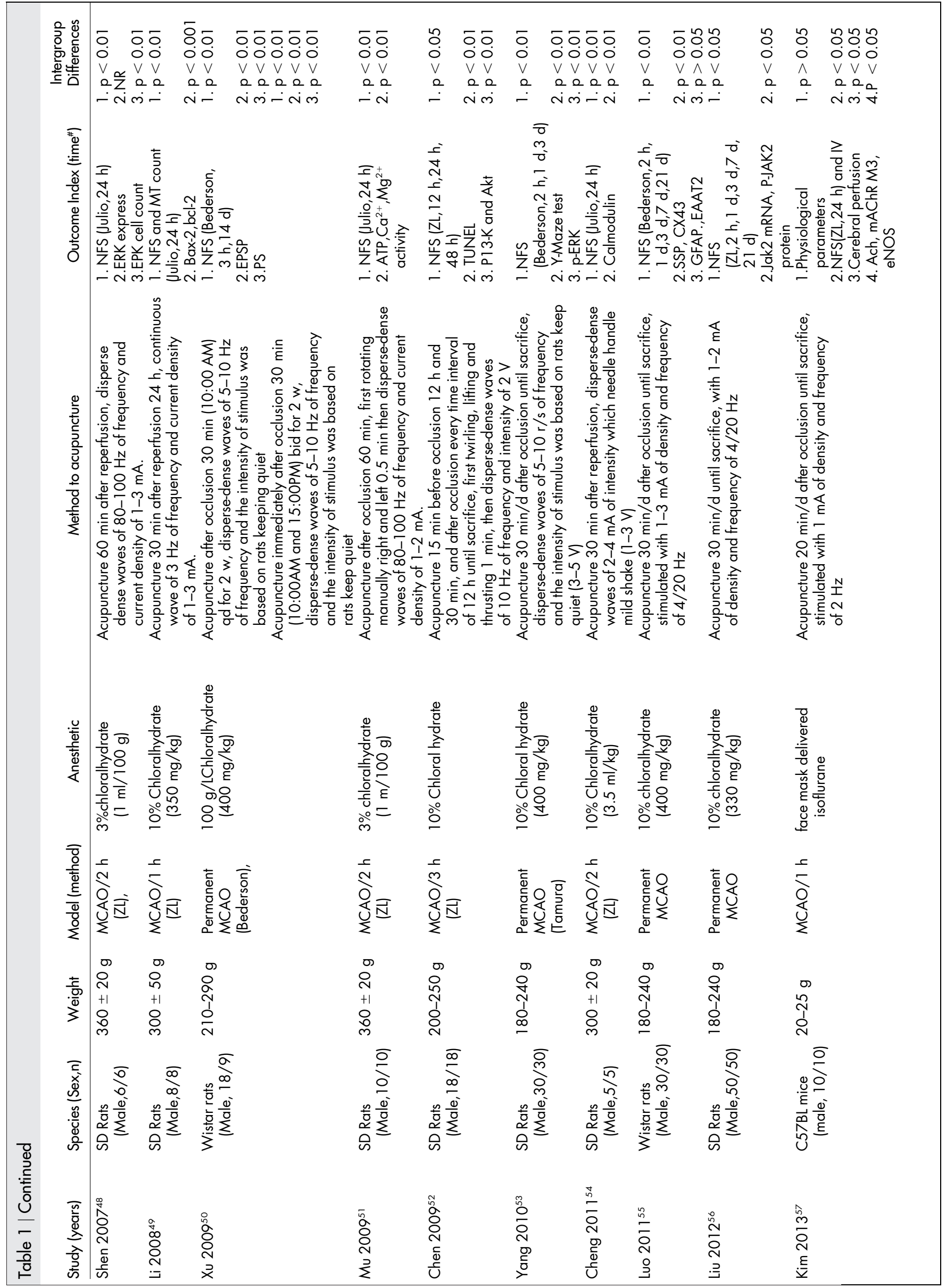




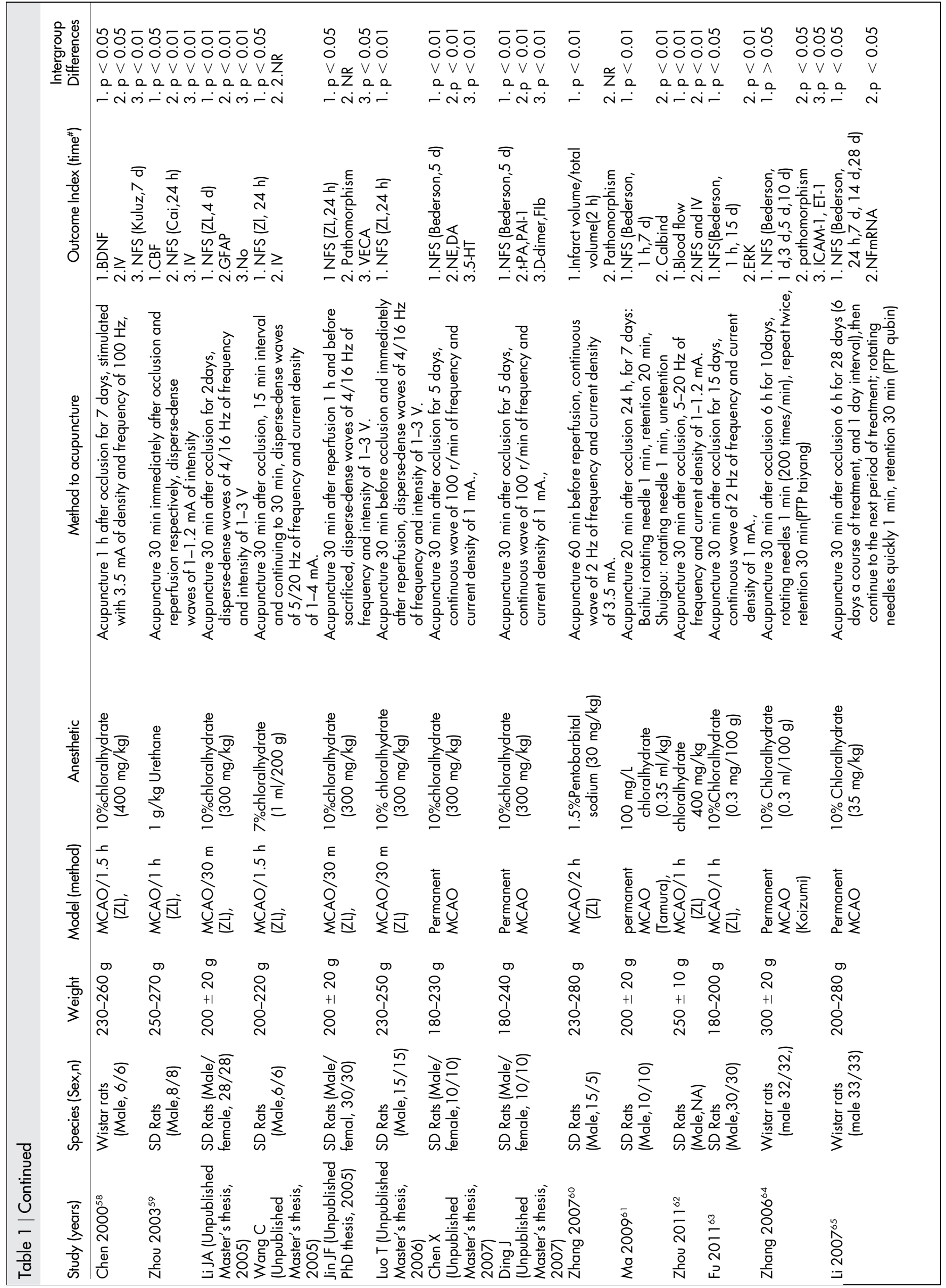




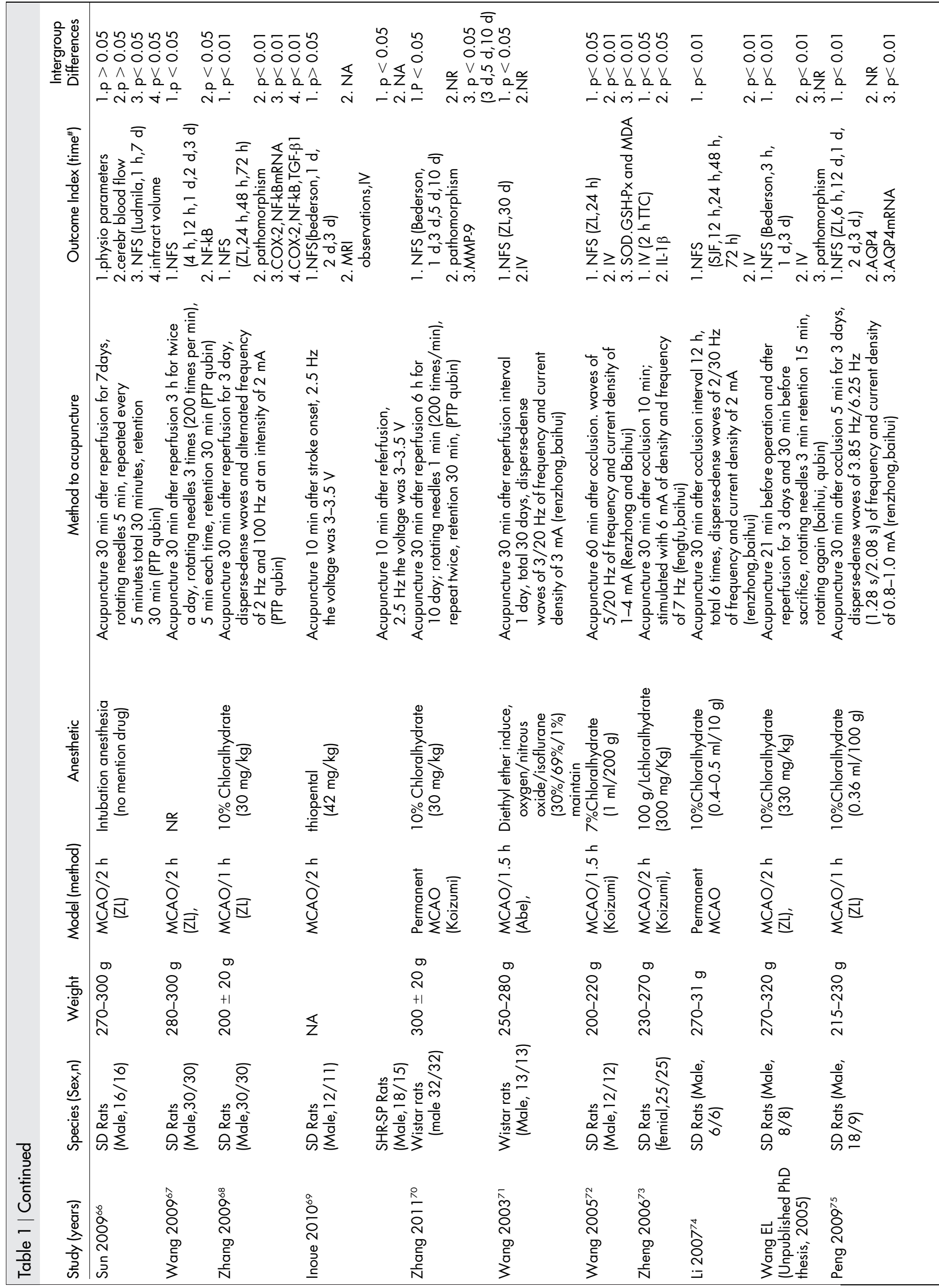




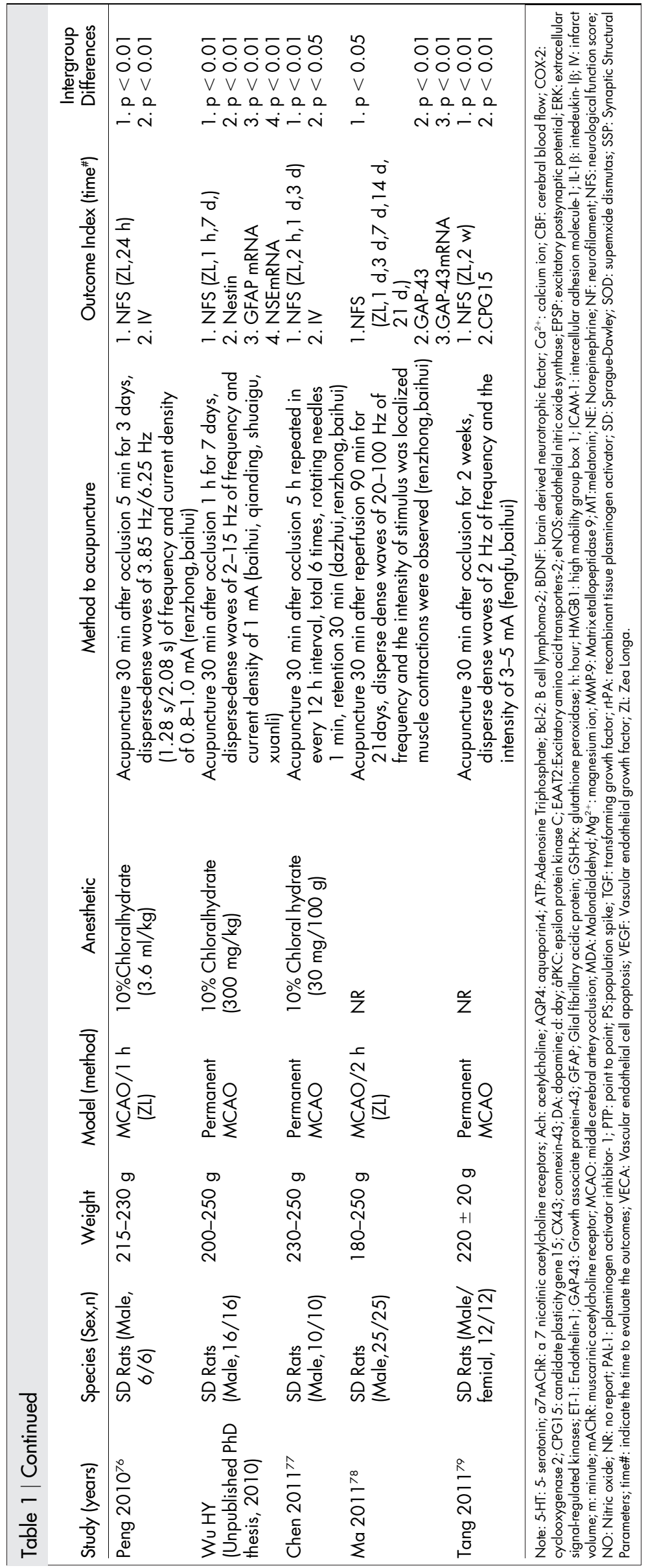




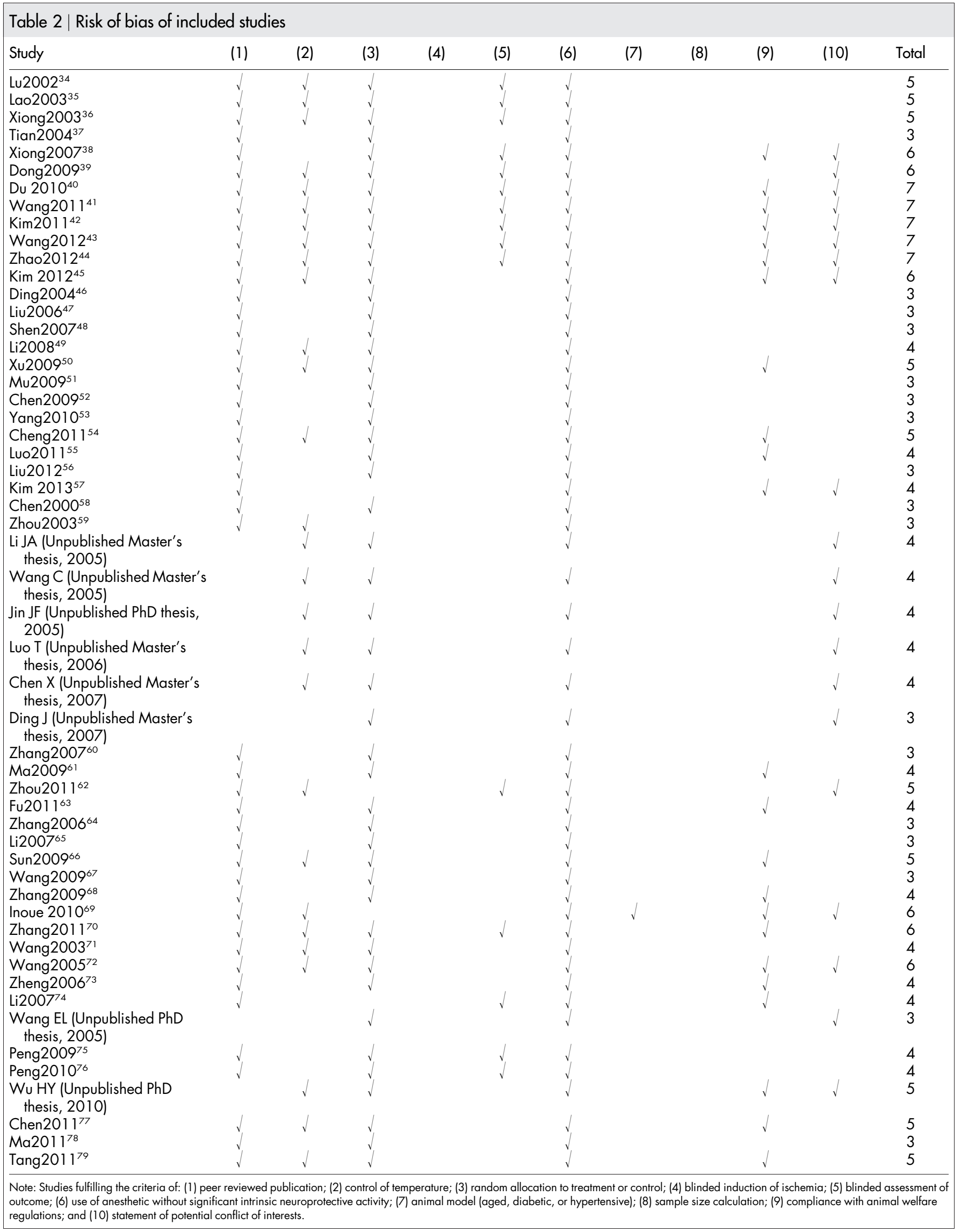




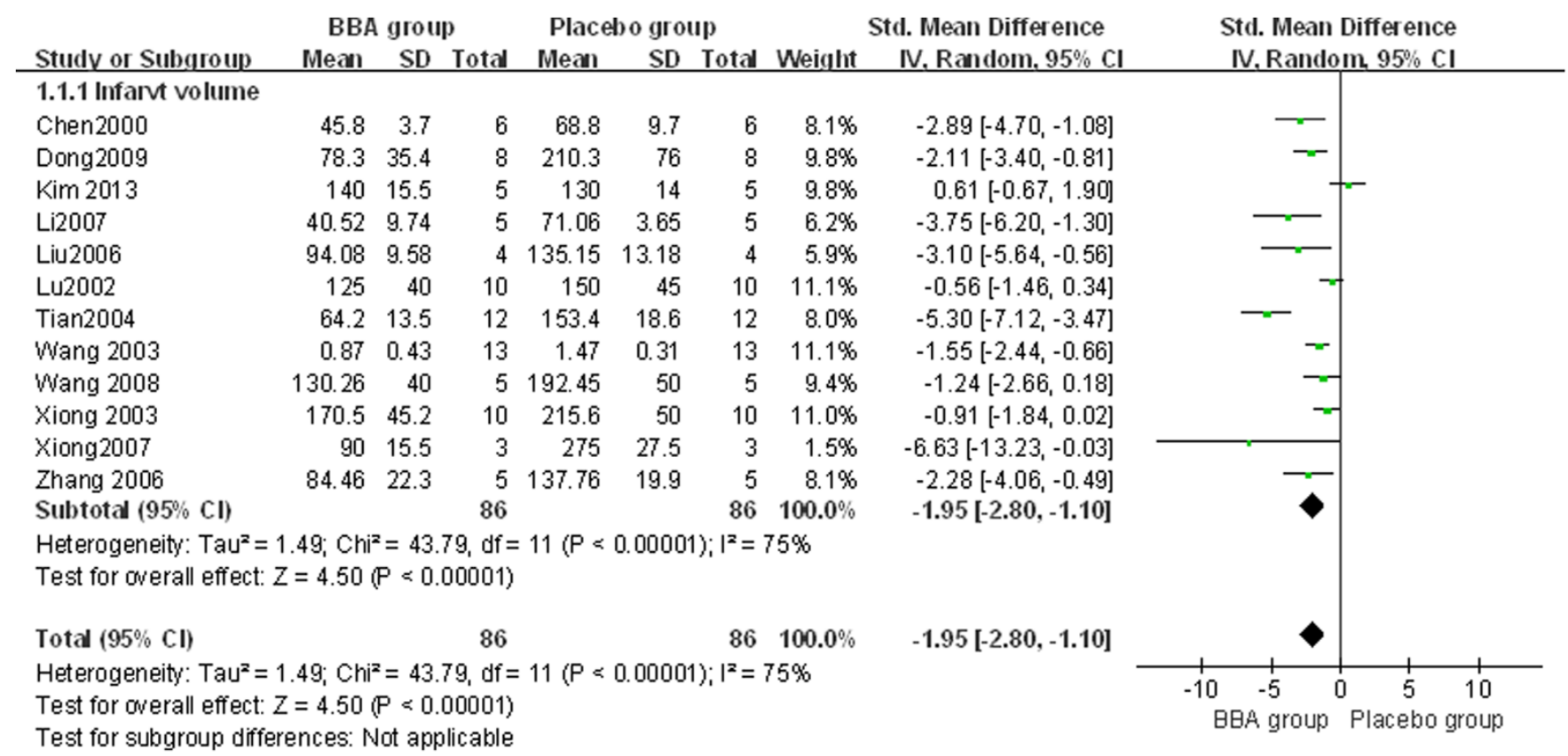

Figure $2 \mid$ Pooled estimate of decrement in infarct volume with Baihui(GV20)-based Scalp acupuncture.

heterogeneity $\chi^{2}=27.21, \mathrm{p}=0.0001, \mathrm{I} 2=78 \%$, Figure $8 \mathrm{C}$ ). Treatment in the published studies also was more effective of BBA in the reduction of IV than that in un-published studies (SMD -2.05 , 95\% CI: $-2.99 \sim-1.12, \mathrm{p}<0.0001$; heterogeneity $\chi 2=43.69, \mathrm{p}<$ $0.00001, \mathrm{I}^{2}=77 \%$, Figure $\left.8 \mathrm{D}\right)$. These results were consistent with previous subgroup analysis of NFS except time interval from the onset of ischemia.

\section{Discussion}

Efficacy of BBA. To our knowledge, this is the first systematic review and meta-analysis of English and Chinese literatures to determine the efficacy of BBA for animal model of acute ischemic stroke with IV and NFS as the outcome measures. The present study indicated that
BBA could substantially improve neurobehavioral deficits and reduce infarct size in animal model of focal cerebral ischemia, suggesting that BBA have potential neuroprotective role in acute ischemic stroke.

Methodological considerations. This systematic review has a number of weaknesses. First, our search did not include data in other languages except Chinese and English, which may result in certain degree of selective bias. Second, negative studies are less likely to be published, and some negative results could not be obtained. In the present study, treatment in the published studies was more effective than that in un-published studies. Thus, the effect may be overestimated. Third, methodological quality of the included

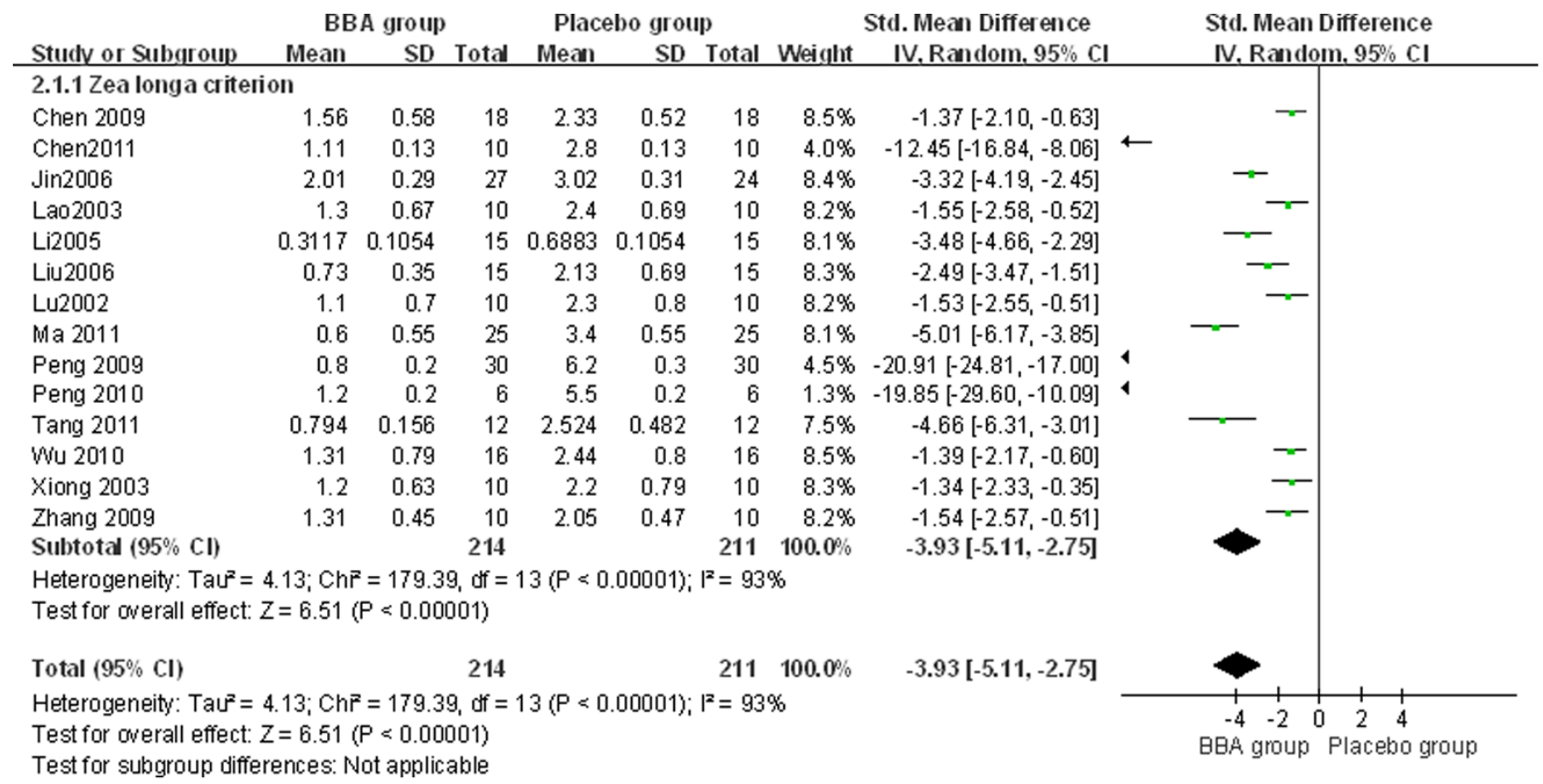

Figure 3 Pooled estimate of improvement in neurological function score with Baihui(GV20)-based Scalp acupuncture according to Zea longa criteria. 


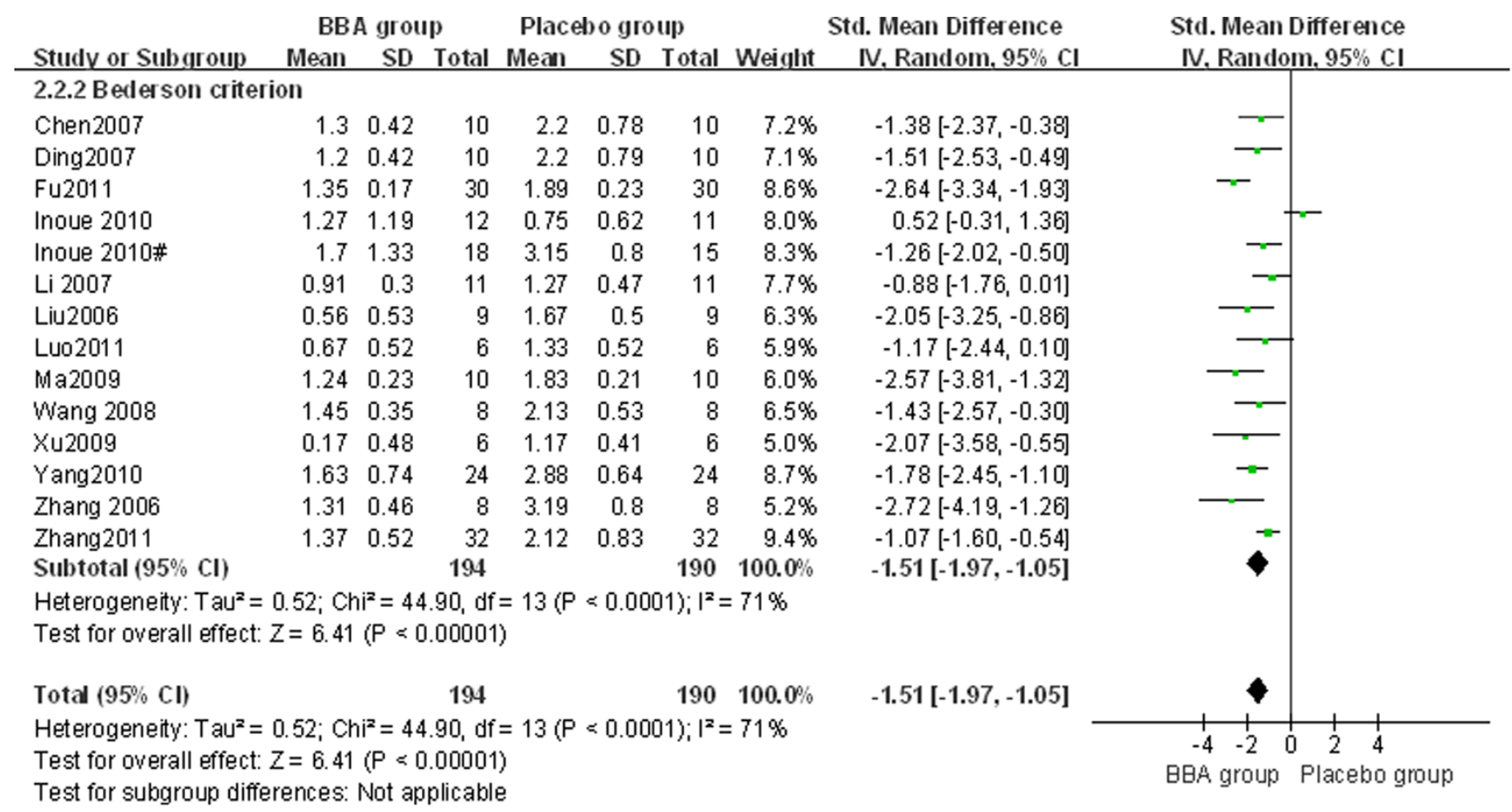

Figure 4 | Pooled estimate of improvement in neurological function score with Baihui(GV20)-based Scalp acupuncture based on Bederson criteria.

studies was generally low, which is an inherent limitation in the primary study. Quality of a study has significant impacts on the reported outcome. For example, reporting randomization and blinding are less likely to report positive findings than those neither ${ }^{84}$. Low quality of the included studies suggested that the results should be interpreted with caution.

Implication for further studies. There are various advantages of EA as it is more readily controlled, standardized and objectively measurable than manual acupuncture. In some situations EA was more effective than traditional acupuncture, particularly when strong, continued stimulation is required, as when treating paralysis ${ }^{85}$. EA was also recommended for clinical trials and mechanism researches on acupuncture ${ }^{86}$. In the present study, 46 out of 54 studies performed EA. Thus, EA have priority over Manual acupuncture on acupuncture research for the animal models of stroke.

In animal model of focal cerebral ischemia, the multitudinous pathophysiological processes which are involved in their deleterious effects over different time courses extending from the first hours to several days after vessel occlusion ${ }^{87}$. In the present study, efficacy of BBA was lower in MCAO 3 hours and permanent groups compared with other temporary ischemia groups, thus it might be inferred that BBA could effectively inhibit those pathophysiological pathways preferentially activated by reperfusion. Further studies would be required to evaluate when the optimum time window for BBA treatment would close and to determine the duration of time to achieve maximum efficacy.

According to the effect size, this study indicated that SD rats recovered better than Wistar rats. A hypothesis may arise that individual genetic differences have different neuroprotective role in ischemic stroke. A comparison among anesthetics showed more effectiveness in NFS improvement in studies using chloral hydrate than studies using other two anesthetics. Thus, future study design for animal research need to select suitable anesthetics.

In the present study, most animal models of stroke are established on normotensive animals with occlusion of cerebral artery to artificially induce infarction in brain. The relevance of animal models

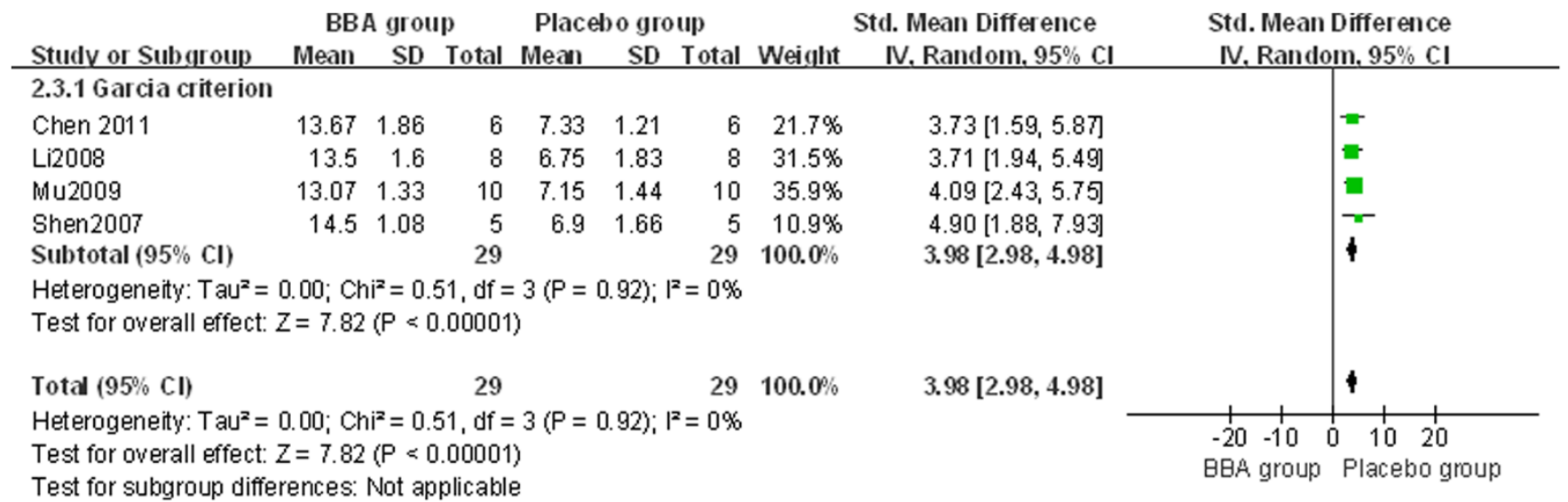

Figure 5 | Pooled estimate of improvement in neurological function score with Baihui(GV20)-based Scalp acupuncture based on Garcia criteria. 


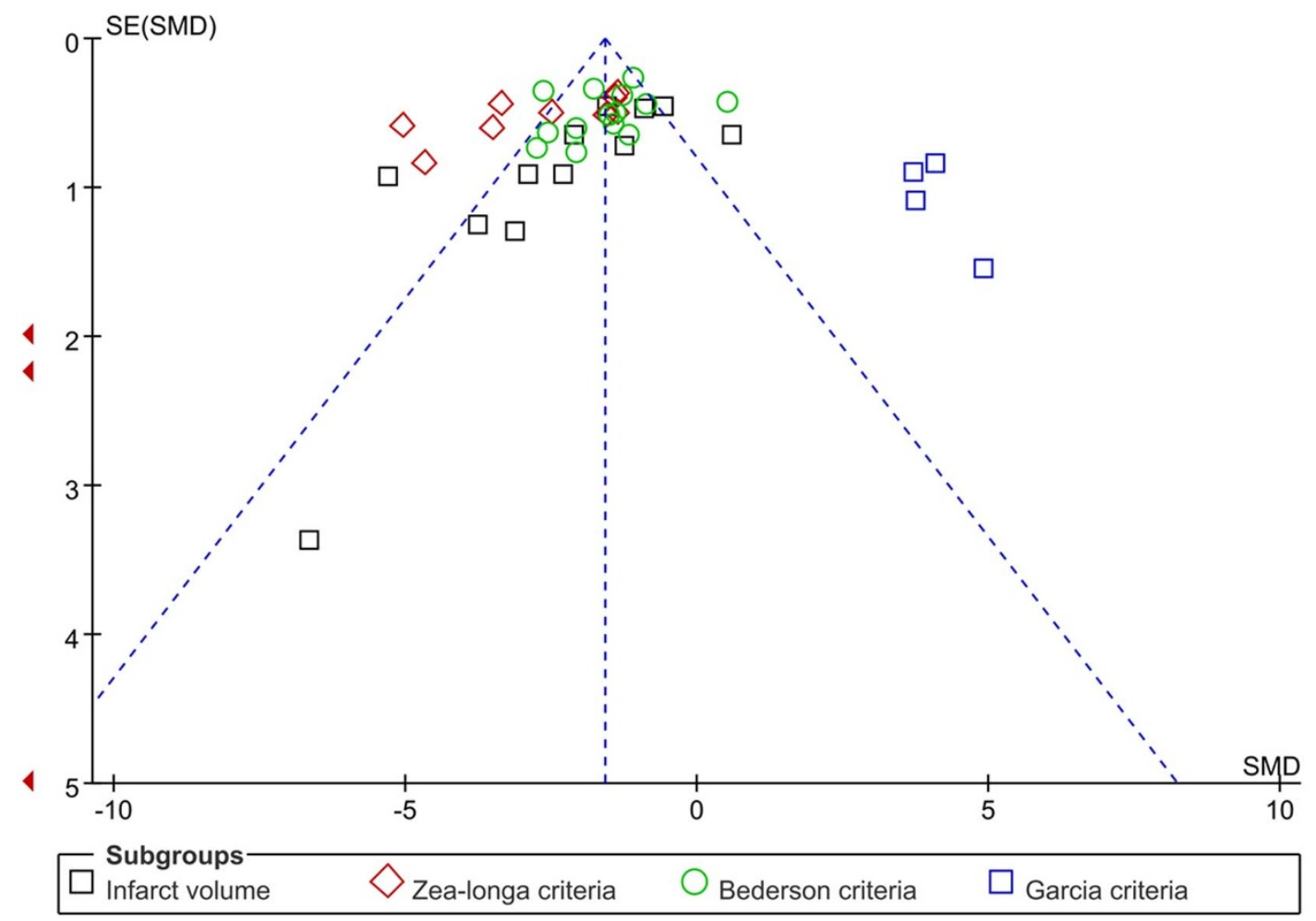

Figure 6 Bias assessment plot for the effect of Baihui(GV20)-based Scalp acupuncture on infarct volume and neurological function score.
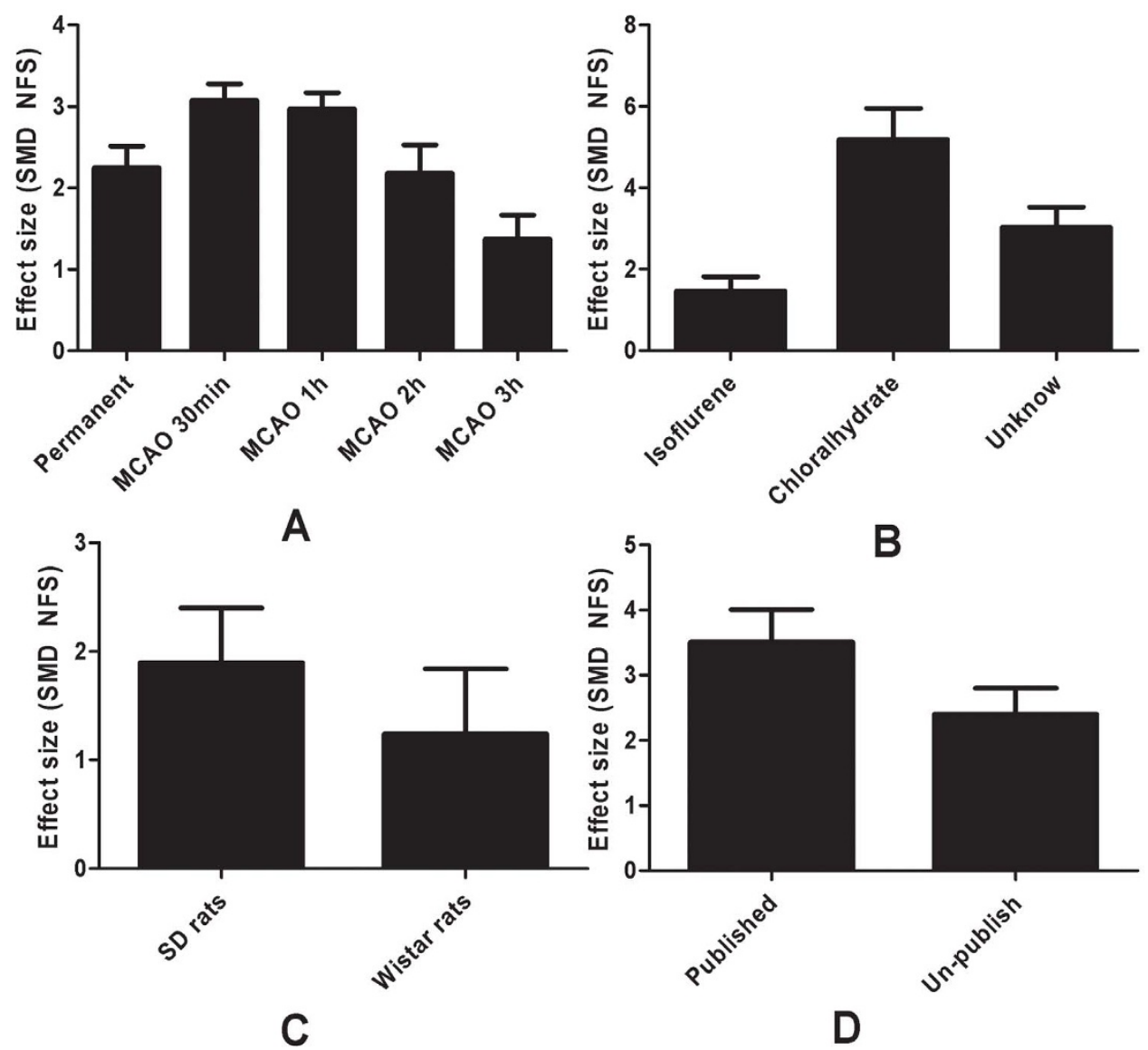

Figure 7 Subgroup analysis according to neurological function score (NFS). (A) The effect of the use of model on the estimate of improvement in NFS outcome; (B) The type of anesthetic on the estimate of improvement in NFS; (C) The type of strain used on the estimate of improvement in NFS; (D)The impact of published studies comparing with un-published studies on the estimate of improvement in NFS outcome. The vertical error bars represent the effect size for the individual estimates. 

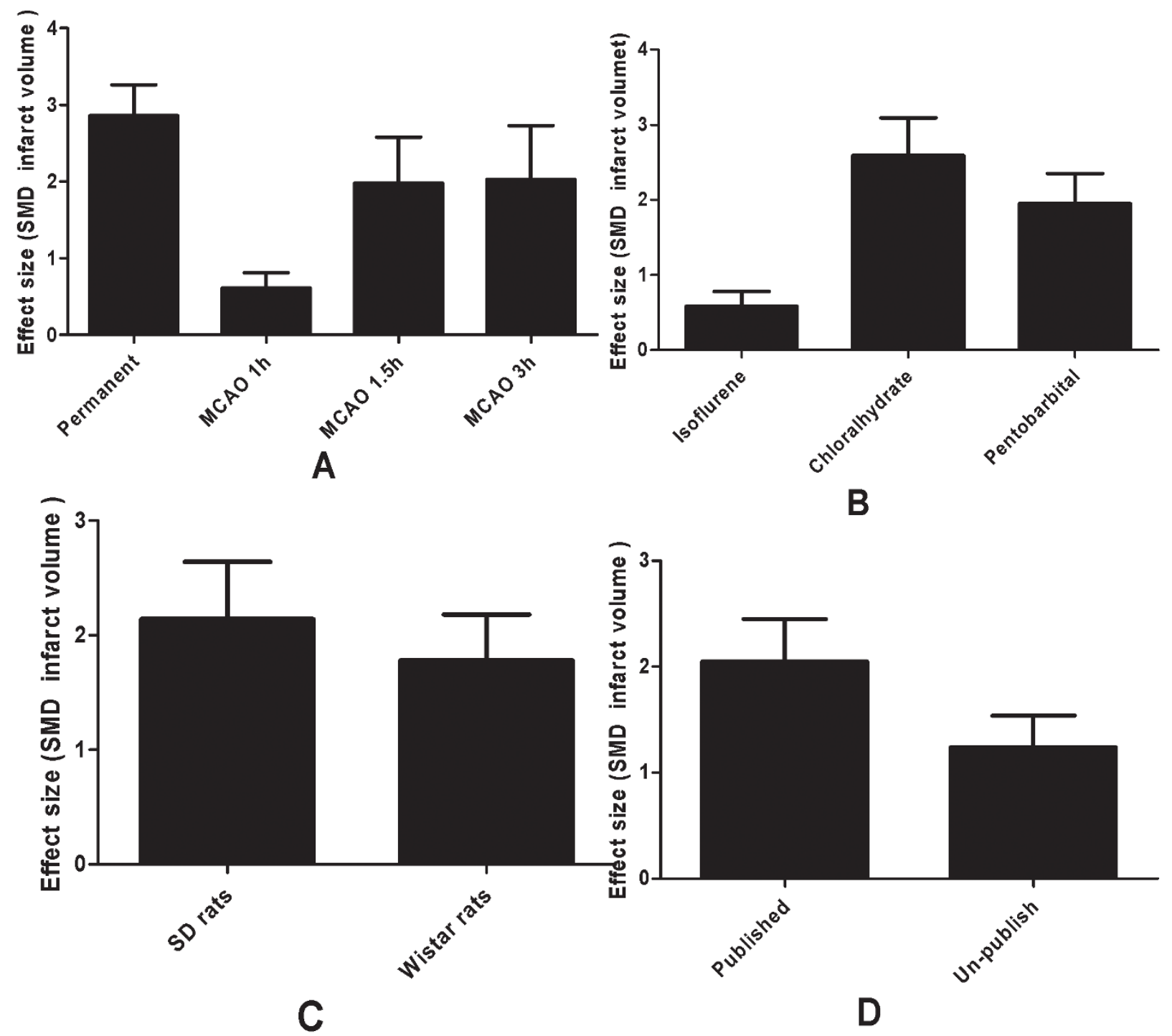

Figure 8 Subgroup analysis according to infarct volume (IV). (A) The effect of the use of model on the estimate of improvement in IV outcome; (B) The type of anesthetic on the estimate of improvement in IV outcome; (C) The type of strain used on the estimate of improvement in IV outcome; (D)The impact of published studies comparing with un-published studies on the estimate of improvement in IV outcome. The vertical error bars represent the effect size for the individual estimates.

with normal cerebrovascular structure to human conditions remains dubious $^{88}$. Impressively, Scalp acupuncture had a rapid and strong effect on neurological dysfunction only in the hypertensive strokemodel by reducing the vasogenic oedema, but had no significant effects on the cytotoxic oedema, vasogenic oedema or neurological dysfunction of the MCAO rats within the time span examined ${ }^{89}$. Hence, future studies need to investigate EA efficacy in animals with a co-morbidity such as hypertension, diabetes or advanced age.

\section{Conclusion}

In animal model of focal cerebral ischemia, BBA could improve IV and NFS. Although some factors such as study quality and possible publication bias may undermine the validity of positive findings, BBA may have potential neuroprotective role in experimental stroke.

\section{Methods}

Search strategy. We identified studies of BBA in animal models of acute ischemic stroke from Cochrane Central Register of Controlled Trials (CENTRAL), PubMed, EMBASE, Chinese National Knowledge Infrastructure (CNKI), VIP information database, and Wanfang data Information Site. The publication time is from the inception of each database up to June 2013. The search term used was "Baihui (GV20)" in English or in Chinese. All searches were limited to studies on animals.
Reference lists from the included literature were used to identify further relevant publications.

Eligibility. We included all controlled studies of the effect of BBA in animal models of focal cerebral ischaemia, where the outcome was measured as infarct volume (IV) or/ and neurological function score (NFS) ${ }^{27}$. IV is an essential indicator of the severity of brain ischemic injury. 2,3,5-triphenyltetrazolium chloride (TTC) staining is an excellent research method that can be used to confirm the size and location of areas of infarction induced by focal cerebral ischemia in rats ${ }^{28}$. Briefly, brain IV was assessed by TTC staining for 10 minutes at $37^{\circ} \mathrm{C}$, followed by overnight immersion in $4 \%$ paraformaldehyd; unstained areas were defined as IV. NFS can also be useful in animal studies that evaluate the effect of new therapeutic methods; moreover, physical testing of the animals can be repeated over time and thus provide data on the evolution of the neurological deficit. However, measuring methods of NFS were inconsistent in different studies. Three neurological grading systems were most commonly used. The first grading system was published by Bederson et $\mathrm{al}^{29}$. in 1986. This system consists of a scale from 0 to 3: (0) no observable deficit; (1) decreased forelimb resistance to a lateral push; (2) forelimb flexion; (3) circling behavior in addition to the former symptoms. The second system was reported by Zea Longa et $\mathrm{al}^{30}$. in 1989 . The scale rates the presence or absence of neurological signs in rats, and the details are as follows: $0^{\prime}$-no neurological deficit; $1^{\prime}$-retracts left forepaw when lifted by the tail; $2^{\prime}$-circles to the left; $3^{\prime}$-falls while walking; $4^{\prime}$-does not walk spontaneously; $5^{\prime}$-dead after surgery. The third system was introduced by Garcia et $\mathrm{al}^{31}$. in 1995. It consists of 6 different criteria, including spontaneous activity, symmetry in the movement of the 4 limbs, forepaw outstretching, climbing, body proprioception, and response to vibrissae touch. The individual performance in each 
test was rated on a 0 to 3 point subscore. The sum of all 6 individual subscores was then calculated to give a range of 3-18. Thus, the score in healthy rats would be 18 .

To prevent bias, inclusion criteria were pre-specified as follows: (1) the effect of BBA was tested on an animal model of focal cerebral ischemia induced by temporary middle cerebral artery occlusion (MCAO) or permanent MCAO; (2) IV and/or NFS were compared with control animals receiving vehicle or no treatment. Pre-specified exclusion criteria were: (1) non-focal cerebral ischemia model (such as global, traumatic models, or hypoxic-ischemic models); (2) combined use of BBA and body acupuncture or ear acupuncture or any other agent with potentially neuroprotective effects; (3) no control group; (4) duplicate publications.

Data extraction. The following details were extracted from each study: (1) publication year and the first author's name, model of ischemic stroke (transient, or permanent), and ischemic time; (2) individual data were obtained for each study, including aimal number, species, sex, weight, motor impairment and scale; (3) information on treatment was obtained, including timing for initial treatment, types and method of treatment procedure, and duration of treatment; (4) outcome measures and timing for outcomes assessments were also included. IV and/or NFS were especially extracted separately. If outcomes were presented from the studies of animals at different time points, we extracted data from the last time point. If the data for meta-analysis were missing or only expressed graphically, we tried to contact the authors for further information, or calculate by ourselves if the information needed were available, or excluded the study which we could not get enough information. For each comparison, we extracted data of mean value and standard deviation from each treatment and control group of every study.

Quality assessment. We evaluated the methodological quality of the included studies by applying a ten-item modified scale ${ }^{32}:$ (1) publication in a peer-reviewed journal; (2) statements describing control of temperature; (3) randomization to treatment group; (4) allocation concealment; (5)blinded assessment of outcome; (6) avoidance of anesthetics with known marked intrinsic neuroprotective properties; (7) use of animals with relevant comorbidities; (8) sample size calculation; (9) compliance with animal welfare regulations; (10) declared any potential conflict of interest. For the calculation of an aggregate quality score, each item of the ten-item modified scale was attributed one point. Two authors (WWW, XCL) independently extracted data and assessed study quality. Disagreements were solved after discussion on the details of the studies.

Statistical analysis. We considered all NFS and IV as continuous data, and then an estimate of the combined effect sizes utilizing standard mean difference (SMD) with the random effects model was given. We used the random effects model rather than the fixed effects model because heterogeneity between multi-studies has to be taken into account. Publication bias was assessed with a funnel plot $^{33}$. For the assessment of heterogeneity, the $\mathrm{I}^{2}$ statistic was used.

Furthermore, to explore the impact of factors modifying on the outcome measures, we performed a stratified meta-analysis with experiments grouped according to the following: duration from onset of ischemia to treatment, anaesthetic method used, the experimental data formally published or not, and species of animals used. Difference between $n$ groups was assessed by partitioning heterogeneity and using the $\chi 2$ distribution with $n-1$ degrees of freedom (df), where $n$ equals the number of groups. All analysises were performed with Revman version 5.1. Probability value $\mathrm{p}<0.05$ were considered significant.

1. Vickers, A. \& Zollman, C. ABC of complementary medicine. Acupuncture. BMJ 319, 973-976 (1999).

2. NIH Consensus Conference. Acupuncture. JAMA 280, 1518-1524 (1998).

3. Wang, Y., Fan, Y. C., Xie, C. L. \& Zheng, G. Q. History of post-stroke epilepsy in ancient China. J neurol 258, 1555-1558 (2011).

4. Zhao, X. F., Du, Y., Liu, P. G. \& Wang, S. Acupuncture for stroke: evidence of effectiveness, safety, and cost from systematic reviews. Top Stroke Rehabil 19, 226-233 (2012).

5. Strong, K., Mathers, C. \& Bonita, R. Preventing stroke: saving lives around the world. Lancet neurol 6, 182-187 (2007).

6. Jauch, E. C. et al. Guidelines for the early management of patients with acute ischemic stroke: a guideline for healthcare professionals from the American Heart Association/American Stroke Association. Stroke 44, 870-947 (2013).

7. World Health Organization (WHO), Guidelines on Basic Training and Safety in Acupuncture 1999.

8. Shen, E. Y., Chen, F. J., Chen, Y. Y. \& Lin, M. F. Locating the Acupoint Baihui (GV20) Beneath the Cerebral Cortex with MRI Reconstructed 3D Neuroimages. Evid Based Complement Alternat Med 2011, 362494 (2011).

9. Cheong, Y. C, Dix, S., Hung Yu Ng, E., Ledger, W. L. \& Farquhar, C. Acupuncture and assisted reproductive technology. Cochrane Database Syst Rev. 2013, Issue 7. Art. No.: CD006920. DOI: 10.1002/14651858.CD006920.pub3. Available at http://summaries.cochrane.org/CD006920/acupuncture-and-assistedconception\#sthash.c460BNCR.dpuf, Assessed as up to date: July 22, 2013.

10. Liu, Z. et al. History and mechanism for treatment of intracerebral hemorrhage with scalp acupuncture. Evid Based Complement Alternat Med 2012, 895032 (2012).
11. Liu, J. H., Bao, C. L., Zhu, W. Z., Zhang, G. B. \& Dong, G. R. Observation on specificity of acupuncture location in treatment of acute apoplexy by scalp penetration needling. Chin Acupunct Moxibust 30, 275-278 (2010).

12. Xu, J., Zhang, B., Zhang, Q. C. \& Xin, S. C. Research on the Status quo of ischemic cerebrovascular disease treated by needling Baihui (DU20) through Qubin (GB7). J Shanxi Coll Tradit Chin Med 10, 73-75 (2009).

13. Inoue, I., Chen, L., Zhou, L., Zeng, X. \& Wang, H. Reproduction of scalp acupuncture therapy on strokes in the model rats, spontaneous hypertensive ratsstroke prone (SHR-SP). Neurosci Lett 333, 191-194 (2002).

14. Dong, H. et al. Repeated electroacupuncture preconditioning attenuates matrix metalloproteinase- 9 expression and activity after focal cerebral ischemia in rats. Neurol Res 31, 853-858 (2009).

15. Kim, J. H. et al. Electroacupuncture acutely improves cerebral blood flow and attenuates moderate ischemic injury via an endothelial mechanism in mice. PloS one 8 , e56736 (2013).

16. Wang, Q. et al. Electroacupuncture pretreatment attenuates cerebral ischemic injury through alpha7 nicotinic acetylcholine receptor-mediated inhibition of high-mobility group box 1 release in rats. I Neuroinflammation 9, 24 (2012).

17. Fang, Z., Ning, J., Xiong, C. \& Shulin, Y. Effects of Electroacupuncture at Head Points on the Function of Cerebral Motor Areas in Stroke Patients: A PET Study. Evid Based Complement Alternat Med 2012, 902413 (2012).

18. Hwang, I. K. et al. Effects of electroacupuncture at Zusanli and Baihui on brainderived neurotrophic factor and cyclic AMP response element-binding protein in the hippocampal dentate gyrus. J Vet Med Sci 72, 1431-1436 (2010).

19. Park, J., Hopwood, V., White, A. R. \& Ernst, E. Effectiveness of acupuncture for stroke: a systematic review. J neurol 248, 558-563 (2001).

20. Sze, F. K., Wong, E., Or, K. K., Lau, J. \& Woo, J. Does acupuncture improve motor recovery after stroke? A meta-analysis of randomized controlled trials. Stroke 33, 2604-2619 (2002).

21. Wu, X. L., Li, X. D., Liu, A. G., Li, Y. Q. \& An, Z. H. Effects of acupuncture on rehabilitation of nervous functions in the stroke patient of different OCSP types. Chin Acupunct Moxibust 28, 328-330 (2008).

22. Kong, J. C., Lee, M. S., Shin, B. C., Song, Y. S. \& Ernst, E. Acupuncture for functional recovery after stroke: a systematic review of sham-controlled randomized clinical trials. CMAJ 182, 1723-1729 (2010).

23. Wu, P., Mills, E., Moher, D. \& Seely, D. Acupuncture in poststroke rehabilitation: a systematic review and meta-analysis of randomized trials. Stroke 41, e171-179 (2010).

24. Wang, Y. et al. Scalp acupuncture for acute ischemic stroke: a meta-analysis of randomized controlled trials. Evid Based Complement Alternat Med 2012, 480950 (2012).

25. Lee, H. S. et al. Scalp acupuncture for Parkinson's disease: a systematic review of randomized controlled trials. Chin J Integr Med 19, 297-306 (2013).

26. Murphy, S. P. \& Murphy, A. N. Pre-clinical systematic review. J Neurochem 115, 805 (2010).

27. Macleod, M. R., O'Collins, T., Horky, L. L., Howells, D. W. \& Donnan, G. A Systematic review and metaanalysis of the efficacy of FK506 in experimental stroke. J Cereb Blood Flow Metab 25, 713-721 (2005).

28. Bederson, J. B., Pitts, L. H., Germano, S. M. \& Bartkowski, H. M. Evaluation of 2,3,5-triphenyltetrazolium chloride as a stain for detection and quantification of experimental cerebral infarction in rats. Stroke 17, 1304-1308 (1986).

29. Bederson, J. B. et al. Rat middle cerebral artery occlusion: evaluation of the model and development of a neurologic examination. Stroke 17, 472-476 (1986).

30. Longa, E. Z., Weinstein, P. R., Carlson, S. \& Cummins, R. Reversible middle cerebral artery occlusion without craniectomy in rats. Stroke 20, 84-91 (1989).

31. Garcia, J. H., Wagner, S., Liu, K. F. \& Hu, X. J. Neurological deficit and extent of neuronal necrosis attributable to middle cerebral artery occlusion in rats. Statistical validation. Stroke 26, 627-634; discussion 635 (1995).

32. Macleod, M. R., O’Collins, T., Howells, D. W. \& Donnan, G. A. Pooling of animal experimental data reveals influence of study design and publication bias. Stroke 35, 1203-1208 (2004).

33. Egger, M, Davey Smith, G., Schneider, M. \& Minder, C. Bias in meta-analysis detected by a simple, graphical test. BMJ 315, 629-634 (1997).

34. Lu, Z. H. et al. Acupoint specificity of electroacupuncture preconditioning effect on cerebral ischemia injury in rats. Chin Acupunct Moxibust 10, 671-673 (2002)

35. Lao, N. et al. Baihui electroacupuncture in the induction of brain ischemic tolerance by preconditioning. J Fourth Military Med Univ 24, 418-420 (2003).

36. Xiong, L. Z. et al. Pretreatment with repeated electroacupuncture attenuates transient focal cerebral ischemic injury in rats. Chin Med J (Engl) 116, 108-111 (2003).

37. Tian, D. S., Deng, Y. Y., Wang, G. H., Guo, G. J. \& Ru, L. Q. Role of electroacupuncture preconditioning against neuronal apoptosis in rats with focal cerebral ischemia. J Fourth Military Med Univ 25, 27-29 (2004).

38. Xiong, L. Z., Yang, J., Wang, Q. \& Lu, Z. H. Involvement of $\delta$-and $\mu$-opioid receptors in the delayed cerebral ischemic tolerance induced by repeated electroacupuncture preconditioning in rats. Chin Med J (Engl) 120, 394-399 (2007).

39. Dong, H. et al. Repeated electroacupuncture preconditioning attenuates matrix metalloproteinase- 9 expression and activity after focal cerebral ischemia in rats. Neurol Res 31, 853-858 (2009).

40. Du, J. et al. Involvement of ERK $1 / 2$ activation in electroacupuncture pretreatment via cannabinoid CB1 receptor in rats. Brain Res 1360, 1-7 (2010). 
41. Wang, Q. et al. Activation of epsilon protein kinase C-Mediated anti-Apoptosis is involved in rapid tolerance induced by electroacupuncture pretreatment through cannabinoid receptor type 1. Stroke 42, 389-396 (2011).

42. Kim, M. W. et al. Scalp electroacupuncture at the Baihui acupoint (DU 20) improves functional recovery in rats with cerebral ischemia. Neural Regen Res 6, 2822-2828 (2011)

43. Wang, Q. et al. Electroacupuncture pretreatment attenuates cerebral ischemic injury through $\alpha 7$ nicotinic acetylcholine receptor-mediated inhibition of high-mobility group box 1 release in rats. J Neuroinflammation 9, 24 (2012)

44. Zhao, Y. et al. Electroacupuncture pretreatment induces tolerance against focal cerebral ischemia through activation of canonical Notch pathway. BMC Neurosci 13, $111(2012)$

45. Kim, M. W. et al. Electroacupuncture enhances motor recovery performance with brain-derived neurotrophic factor expression in rats with cerebral infarction. Acupunct Med 30, 222-226 (2012).

46. Ding, J. et al. Electroacupuncture preconditioning in treatment of focal cerebral ischemia in rats. Chin J Clin Rehabil 8, 106-107 (2004).

47. Liu, J. \& Lai, X. S. Electroacupuncture effect on neurological behavior and infarct volume in SD rats with focal cerebral ischemia. Chin J Basic Med Tradit Chin Med 126, 454-456 (2006)

48. Shen, M. H., Li, Z. R., Xiang, X. R., Niu, W. M. \& Zhang, C. B. Involvement of extracellular regulated protein kinase signal transduction pathway in electroacupuncture-induced improvement of ischemic cerebral injury in rats with cerebral ischemia reperfusion. Acupunct Res 32, 368-372 (2007).

49. Li, Z. R., Shen, M. H. \& Niu, W. M. Involvement of melatonin in the adjusting effect of electroacupuncture in resisting oxygen stress in cerebral ischemiareperfusion injury rats. Acupunct Res 33, 164-168 (2008).

50. Chen, D. F., Lai, Z., Zhang, S. J., Yao, C. K. \& Lin, W. J. Effects of electroacupuncture on $\mathrm{PD}-\mathrm{K} / \mathrm{Akt}$ path in reperfused local cerebral ischemia rats. Chin J Rehabil Med 24, 502-504 (2009).

51. Mu, Y. Y., Li, Z. R., Niu, W. M. \& Shen, M. H. Effect of electrical acupuncture on APT activity of hippocampus mitochondrion of rats with ischemic reperfusion cerebral injury. J Nanjing Univ Tradit Chin Med 25, 268-270 (2009).

52. Xu, Z. H., Xu, N. G., Yi, W., Fu, W. B. \& Jin, R. Effect of Acupuncture at difllerent doses on synaptic plasticity of rats after cerebral ischemia.J Guangzhou Univ Tradit Chin Med 26, 32-37 (2009).

53. Yang, Z. H., Xu, N. G., Yi, W., Yu, T. \& Dong, Z. N. Research of Intervention with Electroacupuncture through regulating MAPK/ERK pathway for cerebral ischemia rats. J Guangzhou Univ Tradit Chin Med 27, 23-26 (2010).

54. Cheng, J. et al. Effects of electroaeupuncture on expression of calmodulin in the hippocampus of rats with cerebral ischemia-reperfusion injury. Chin Acupunct Moxibust 31, 1015-1019 (2011).

55. Luo, Y., Xu, N. G., Yi, W., Yu, T. \& Yang, Z. H. Study on the correlation between Synaptic Reconstruction and Astrocyte after Ischemia and the Influence of Electroacupuncture on Rats. Chin J Integr Med 17, 750-757 (2011).

56. Liu, R., Xu, N. G., Wei, Y., Huang, K. B. \& Su, M. Z. Electroacupuncture effect on neurological behavior and tyrosine kinase-JAK 2 in rats with focal cerebral ischemia. J Tradit Chin Med 32, 1-2 (2012).

57. Kim, J. H. et al. Electroacupuncture acutely improves cerebral blood flow and attenuates moderate ischemic injury via an endothelial mechanism in mice. PLoS One 8, e56736 (2013)

58. Chen, Y. H. \& Huang, X. F. Effect of electroacupuncture on BDNF expression at ischemia cortex and infarct volume after Middle Cerebral Artery Occlusion in Rat. Acupuct Res 25, 165-168 (2000).

59. Zhou, F., Li, F. F., Cheng, J. S. \& Yan, Z. G. Effects of electoracupuncture on regional cerebraI bIood flow and infarction volume in rat model of acute focal cerebral ischemia. Shanghai J Acupunct Moxibust 22, 3-6 (2003).

60. Zhang, T. S. et al. Effect of infarct area and cerebral histopathology with different time course to reperfusion brain of MACO Rats. Chin Arch Tradit Chin Med 25, 1610-1612 (2007).

61. Ma, H. F., Ren, X. J., Lin, C. X. \& Tu, Y. Changes of protein calbindin-D28k expression in hippocampus of cerebral ischemic rats and the influence of acupuncture. J Guangzhou Univ Tradit Chin Med 26, 237-240 (2009).

62. Zhou, F., Guo, J. C., Cheng, J. S., Wu, G. C. \& Xia, Y. Electroacupuncture increased cerebral blood flow and reduced ischemic brain injury: dependence on stimulation intensity and frequency. J Appl Physiol (1985). 111, 1877-1887 (2011).

63. Fu, L., Yi, Y. D., Zhang, T. F. \& Huang, H. Influence of electric acupuncture on ephosphorylation effect of extracellular signal regulated kinase after focal cerebral ischemia in rats brain. Stroke Nerv Dis 18, 6-8 (2011).

64. Zhang, H. M., Fei, Y. T., Shi, Y. J., Jia, B. H. \& Tu, Y. Effects of acupuncture of "Baihui"(GV 20) and "Taiyang" (EX-HN 5) on Functions of Vascular Endothelial Cells in Cerebral Ischemia Injury Rats. Acupuct Res 31, 67-72 (2006).

65. Li, H. Y., Zhu, W. Z., Dong, G. R., Wang, F. J. \& Ke, R. Influence of scale Pointthrough-point acupuncture on $200 \mathrm{kDa}$ neuroflament protein in rats with acute cerebral infarction. Shanghai J Acupunct Moxibust 8, 40-42 (2007).

66. Sun, X. W. et al. Influence of Scale acupuncture on therapy time window in rats with focal cerebral ischemia reperfusion model. Inform Tradit Chin Med 26 66-68 (2009).

67. Wang, G., Sun, X. W. \& Zhou, W. Effects of acupuncture of "Baihui”"(GV 20) Point-through-point "Qubin (G 7)" on neurological function score and NF-xB expression in rats with middle cerebral artery ischemia reperfusion. J Clin Acupunct Moxibust 25, 37-39 (2009).

68. Zhang, H. X. et al. Effects of scalp acupuncture on acute cerebral ischemiareperfusion injury in rats. J Chin Integr Med 7, 769-774 (2009).

69. Inoue, I., Fukunaga, M., Koga, K., Wang, H. D. \& Ishikawa, M. Scalp acupuncture effects of stroke studied with magnetic resonance imaging: different actions in the two stroke model rats. Acupunct Med 27, 155-162 (2009).

70. Zhang, H. M. et al. Effects of scalp acupuncture on expression of hippocampal MMP-9 in cerebral ischemia injury rats. Acupuct Res 36, 193-198 (2011).

71. Wang, S. J. \& Zhang, L. D. ProtectiVe effect of electroacupuncture on the central function in rats with transient middle cerebral artery infarction. Liao Ning J Tradit Chin Med 30, 750-751 (2003)

72. Wang, C. X., Li, Z. R. \& Chen, B. Y. ProtectiVe effect of electroacupuncture on cerebral fhnction via ameliorating oxidative stress in MCAo rats. Neurosci Bull 21, 153-157 (2005).

73. Zheng, Z. Q., Liu, L. J. \& Li, J. H. Changes in cerebral infarction volume and the expression of interleukin 1 beta protein in rats with cerebral ischemic reperfusion after treatment of electroacupuncture. Chin J Clin Rehabil 10, 153-155 (2006)

74. Li, T. L., Yan, J., Chang, X. R. \& Shen, Q. Effect of Electro-acupuncturing Different Points on Behavior Score and Cerebral Infarction Volume of Rats with Acute Cerebral Ischemia. Guiding J TCM 13, 5-8 (2007).

75. Peng, Y. J. et al. Effects of electroacupuncture on AQP4 in rats with cerebral ischemia reperfusion. Guangming J Chin Med 24, 12-15 (2009).

76. Peng, Y. J., Chen, L., Wang, H. S. \& Zhou, F. Therapeutic effects of electroacupuncture at different acupoints in treating acute cerebral ischemia reperfusion in rats. J Anhui Tradit Chin Med Coll 29, 31-33 (2010).

77. Chen, C. T. et al. Effects of Acupuncture on neurologic impaiment and infarct size after local Cerebral ischemia in rats. J Tradit Chin Med Univ Hunan 31, 63-66 (2011).

78. Ma, R. R. et al. Effect of electroacupuncture of different acupoints on GAP-43 expression after focaI cerebraI infarction in rats. J Chongqing Med Univ 36, 38-40 (2011).

79. Tang, X. M. et al. Study on the influences of electroacupuncture at "Baihui, Fengfu” in treating I/R injury rats via regulate hippocampus CPG15 expression. Tradit Chin Med J 10, 60-63 (2011)

80. Kuluz, J. W., Prado, R. J., Dietrich, W. D., Schleien, C. L. \& Watson, B. D. The effect of nitric oxide synthase inhibition on infarct volume after reversible focal cerebral ischemia in conscious rats. Stroke 24, 2023-2029 (1993).

81. Belayev, L., Alonso, O. F., Busto, R., Zhao, W. \& Ginsberg, M. D. Middle cerebral artery occlusion in the rat by intraluminal suture. Neurological and pathological evaluation of an improved model. Stroke 27, 1616-1622; discussion 1623 (1996).

82. Zhang, Y. G., Liu, T. P., Qian, Z. Y. \& Liu, D. Influence of total saponins of panax ginseng oninfarct size and polyamine contents in rat brain after middle cerebral artery ocelussion. Chin J Pharmacol Toxicol 8, 250-255 (1994).

83. Sun, J. F. Methodology of Experimental Animals. 1st edition. (People's Medical Publishing House, Beijing, 2001).

84. Bebarta, V., Luyten, D. \& Heard, K. Emergency medicine animal research: does use of randomization and blinding affect the results? Acad Emerg Med 10, 684-687 (2003)

85. Mayor, D. Electroacupuncture: An introduction and its use for peripheral facial paralysis. J Chin Med 84, 1-17 (2007)

86. Napadow, V. et al. The status and future of acupuncture mechanism research. J Altern Complement Med 14, 861-869 (2008).

87. Dirnagl, U., Iadecola, C. \& Moskowitz, M. A. Pathobiology of ischaemic stroke: an integrated view. Trends Neurosci 22, 391-397 (1999).

88. Wiebers, D. O., Adams, H. P. Jr. \& Whisnant, J. P. Animal models of stroke: are they relevant to human disease? Stroke 21, 1-3 (1990).

89. Inoue, I., Fukunaga, M., Koga, K., Wang, H. D. \& Ishikawa, M. Scalp acupuncture effects of stroke studied with magnetic resonance imaging: different actions in the two stroke model rats. Acupunct Med 27, 155-162 (2009).

\section{Acknowledgments}

This project was supported by the grant of National Natural Science Foundation of China (81173395/H2902); The young and middle-aged university discipline leaders of Zhejiang province, China (2013277)

\section{Author contributions}

All authors have contributed to this article. W.W.W., C.L.X. and G.Q.Z. searched the databases, extracted the data, and screened trials. W.W.W., C.L.X. and L.L. reformed the tables. G.Q.Z., W.W.W. and C.L.X. appraised the quality of included trials and drafted the full text. G.Q.Z. and L.L. were responsible for editing. G.Q.Z. also acted as an arbitrator and conceived the article. All authors reviewed the manuscript.

\section{Additional information}

Competing financial interests: The authors declare no competing financial interests. 
How to cite this article: Wang, W.-W., Xie, C.-l., Lu, L. \& Zheng, G.-q. A systematic review and meta-analysis of Baihui (GV20)-based scalp acupuncture in experimental ischemic stroke. Sci. Rep. 4, 3981; DOI:10.1038/srep03981 (2014). (c) (i) (2) This work is licensed under a Creative Commons AttributionBY ${ }_{\text {NC }}$ SA NonCommercial-ShareAlike 3.0 Unported license. To view a copy of this license, visit http://creativecommons.org/licenses/by-nc-sa/3.0 